\title{
Caminando en la didáctica de la lengua rifeña
}

Jahfar Hassan Yahia

Responsable del seminario Permanente de Lengua y Cultura Tamazight

\section{Presentación}

"Caminando en la didáctica de la lengua rifeña" no pretende ser un manual específico de gramática rifeña; sobre la materia hay elaborados bastantes y muy buenos trabajos. Su concepción es otra. Es la de convertirse en una fuente de recursos didácticos en fichas, con el fin de hacer más fácil y asimilable la comprensión y el aprendizaje de la lengua tamazight. Se pretende de igual modo con ello suscitar la generación de ideas prácticas en el ámbito de la didáctica activa donde el aula y el trabajo de campo se instrumentalizan mutuamente para una mayor optimización de la función enseñanza / aprendizaje. Aunque esto no implica, evidentemente, que se obvie o ignore la gramática, ya que en la mayoría de los casos es imprescindible recurrir a la misma para ilustrar, lo más aproximadamente posible, las acepciones, matices y sentidos semánticos, morfológicos y de sintaxis que expresan y manifiestan tanto la estructura de la oración así como sus elementos. El planteamiento, en síntesis, que nos rige en el presente trabajo es que el aprendizaje del idioma, en el aula, prime sobre el conocimiento de la gramática y en caso de necesidad instrumentalizar ésta última sólo para facilitar el aprendizaje de la lengua.

El muestrario de fichas expuesto en este trabajo forma parte de un conjunto muchísimo mayor que viene elaborándose continuamente y sometiéndose al criterio y observaciones de los participantes del Seminario Permanente de Lengua y Cultura Tamazight.

La propuesta que aparece en la presente con el título Claves de interés para la investigación del contexto amazigh fue elaborada a principios de 1996. Por aquel entonces apenas disponíamos en la ciudad de referencias bibliográficas que nos pudieran enriquecer más en el desarrollo de las ideas expuestas en la citada propuesta. No obstante. la misma sigue vigente sin que hasta la fecha, por motivos sociopolíticos, se hayan logrado gran parte de los fines y objetivos en ella recogidos. 


\section{Introducción}

El presente trabajo, "Claves de interés para la Investigación del contexto amazigh", tiene como objetivo aportar ideas generadoras a un seminario que se presume apasionante, ya que nos pone ante un campo de investigación muy amplio, y tratar una lengua que posee una riqueza lingüística y cultural antiquísima.

La finalidad de este trabajo es el estudio intensivo de la lengua y cultura tamazight en sesiones planificadas, usando fuentes autorizadas de información. Pretende, también en sus planteamientos, el aprendizaje activo pues los participantes en el seminario no acuden, expresamente, para recibir información elaborada, sino que investigan. Numéricamente, el seminario debería oscilar entre cinco y 12 miembros por monitor.

Por los argumentos expuestos seria conveniente que los participantes a admitir en el seminario reuniesen ciertas aptitudes e inquietudes por la cultura y lengua tamazight, porque se trata de una experiencia piloto y en segundo lugar porque el seminario lo caracterizan la actividad, la participación, la elaboración de documentos y conclusiones y la aceptación de estas por los componentes. Es por ello pues por lo que se ha de exigir de los participantes el mayor interés y el máximo de aportación a este seminario.

El presente trabajo abarca los siguientes espacios de investigación:

1. Trabajo de gabinete: Que consiste en recopilar el máximo numero posible de fuentes de información y someterlas a estudio y análisis para posteriormente obtener las conclusiones oportunas.

2. Trabajo de campo: nos permite el contacto directo con el medio amazigh así como la obtención de información de primera mano.

3. Taller de lengua: tiene como objetivo principal, tal como se expone, recuperar del desuso un amplísimo vocabulario beréber, que cada vez se ve mas desplazado. Así mismo enriquecer el fichero lingüistico con las aportaciones de estas sesiones con las mujeres adultas, ya que se puede afirmar que la mujer beréber es una de las razones principales de la vitalidad de la lengua tamazight.

4. Distribución de la información obtenida, analizada y clasificada: consiste primordialmente en; diseñar programas, material didáctico oportuno, perfeccionar métodos docentes específicos para la enseñanza de la lengua tamazight y contribuir a la optimización del aprendizaje de los participantes que se integren en los sucesivos cursos. Indudablemente, para esta primera etapa, se ha de concebir el seminario como una experiencia piloto donde la actividad investigadora ha de tomar un carácter predominante. La investigación científica del contexto amazigh es un trabajo de equipo (participantes, monitores) que precisa de actividad y participación. Es esencial en el seminario la colaboración científica monitor-participante. Correspondiendo al monitor, además de la dirección adecuada del proyecto de investigación, aportar con eficacia y eficiencia sus conocimientos de la lengua y cultura tamazight así como su experiencia en la localización y búsqueda de la información relativa al espacio que nos ocupa. 


\section{Enseñanza-aprendizaje de la lengua tamazight}

En cuanto a la enseñanza-aprendizaje de la lengua tamazight hay que destacar que toda lengua precisa para su enseñanza-aprendizaje partir de la oralidad para después pasar a la escritura para, finalmente, cuando el participante haya alcanzado plenamente el periodo de las operaciones concretas, iniciar unas líneas gramaticales muy elementales y relacionarlas con la oralidad. Esta es la secuenciación que muchos entendidos sugieren para la enseñanza-aprendizaje de una lengua, especialmente, con las características del tamazight.

Para unos participantes con escasos conocimientos de esta lengua o poca soltura oral de la misma, habría que ofrecerles un método que les permitiera la adquisición gradual y efectiva de la lengua tamazight.

El aprendizaje del tamazight sin una base suficiente de conocimientos de la misma lengua, no puede llevar a unos resultados aceptables, por lo que es preciso adoptar un procedimiento en el que el aprendizaje ora/ de la lengua tamazight ocupara un espacio imprescindible.

Esta conclusión nos exige contar con una guía para la enseñanza oral del tamazight. Afortunadamente, disponemos en la ciudad de un material, que en su día fue elaborado y adaptado para el Plan Piloto de Formación Integral Ciudadana de Melilla, que fue diseñado, con la colaboración de los monitores de este plan de formación, para la enseñanza oral del castellano y pensado para los grupos con escasos conocimientos de este idioma.

Este método de oralidad aunque esté diseñado para la enseñanza oral del castellano, eso no impide que sus contenidos y su metodología puedan ser, cómodamente, adaptados por los monitores del seminario a la lengua tamazight, ya que las técnicas didácticas aplicadas y las estructuras gramaticales contenidas en esta guía de oralidad son ajustables a cualquier otra lengua.

Este método se basa, principalmente, en las preguntas. Esto implica que el participante tiene que comprender la pregunta (recepción) y formular la respuesta (oralidad). Se da más importancia a las estructuras que a las palabras sueltas. La ventaja consiste en que la estructura es un instrumento lingüistico con mucha mas capacidad de generación de lenguaje.

Los ejemplos prácticos son la herramienta a la que hay que recurrir para una mejor y más rápida asimilación de la lengua. Es mejor encontrar estructuras básicas a partir de ejemplos, que partir de explicaciones gramaticales, es decir, se ha de hacer más práctica que teoría, ya que las reglas gramaticales se deducen a partir de los ejemplos.

Es evidente, pues, que el método oral inyecta dinamicidad en el aula ya que se introduce la práctica del habla inmediatamente. Asi mismo, este sistema ofrece al monitor la posibilidad de controlar de forma continuada los progresos de los participantes.

Se ha de destacar, en primer lugar, que este manual (Meloral) es un libro de enseñanza-aprendizaje que sirve al monitor ideas y recursos parciales para su trabajo en el aula, y además un esquema válido y experimentado para la enseñanza oral de un idioma. 
El manual pretende ser una guía abierta, es decir flexible a cambios introducidos por el monitor, según las necesidades de la clase diaria.

El manual contiene 31 lecciones, cada lección contiene la introducción de una estructura gramatical. La secuencia de las lecciones sigue el orden lógico de un esquema de enseñanza-aprendizaje de idiomas, muy experimentado. El grado de dificultad, tanto como la densidad en cada lección varía bastante, de manera que existen lecciones mas y menos difíciles.

Las lecciones carecen de una programación temporal, para que el monitor tenga la libertad de programar su procedimiento según los progresos de los participantes.

Este método de oralidad del que se ha hecho la anterior descripción lleva como título (Meloral) y la autora de este trabajo fue la empresa ProEmpleo con sede en Madrid. La publicación de esta guía corrió a cargo del Ministerio de Educación y Ciencias y el Excmo. Ayuntamiento de Melilla.

No cabe duda de que es bastante acertada la opinión de iniciar la enseñanza-aprendizaje de la lengua tamazight partiendo de la oralidad, por tratarse de un método que favorece y facilita, con mayor inmediatez, la generación de lenguaje.

Pero esta opinión, no nos impide tratar, paralelamente a la oralidad, la enseñanza-aprendizaje de la escritura y gramática tamazight. Aunque esto último revista algunas dificultades técnicas ligadas esencialmente a que la lengua tamazight es de tradición oral y que su paso por la escritura continúa en fase de investigación sin que hasta la fecha se haya concluido y consensuado por los investigadores de esta lengua un sistema gráfico de representación definitivo.

Se barajan tres grafías: El tifinagh, los caracteres latinos y los caracteres árabes. En lo que atañe a esto ultimo hay, claro está, muchos puntos de vista. Están los que prefieren escribir en árabe, dado que los imazighen pertenecen a una cultura, intrínsecamente ligada a la lengua árabe, y otros que prefieren volver a las fuentes y rehabilitar la escritura tifinagh. En cambio hay gente que dice: Si queremos acceder al siglo XX, es decir, al siglo de la comunicación, debemos utilizar los caracteres latinos.

De todas formas hay que escribir la lengua tamazight, porque de esto depende su supervivencia y su porvenir, no importa la forma gráfica adoptada, siempre y cuando encaje técnica y eficazmente con las singularidades fonéticas de la lengua tamazight y sus características lingüísticas.

Es importante resaltar, que para pasar a la enseñanza de la lengua tamazight se debe de tener en consideración los libros y manuales realizados, cuando se vallan a tratar los aspectos puramente gramaticales. En este sentido creo conveniente recurrir para la enseñanza-aprendizaje e investigación de la lengua tamazight a los manuales autorizados. La razón es sencilla, difícilmente podemos comenzar a investigar desde la nada. Cuando existe una documentación escrita sobre un tema, debemos recurrir a ella, basándonos en el prestigio de las personas que elaboraron la información.

Podemos, perfectamente, basarnos para nuestro trabajo en la obra del franciscano Pedro Sarrionandía, Gramática de la lengua rifeña, que podemos ver como la obra capital y fundamental de la lingüística colonial española y que coloca a su autor a la cabe- 
za de los berberistas españoles. Este fue el primero que reveló la existencia de un dialecto especial rifeño enclavado como islote lingüístico. En su utilísima Gramática reunió copiosas observaciones sobre la fonética, los accidentes del nombre y del verbo, los artículos y la sintaxis. Gramática fue editada en Tánger en 1905 reeditándose en 1925 , y sirve aún de guía a cuantos tratan de llegar al conocimiento de esta lengua.

Además de la obra anterior, sería de mucho interés recurrir a otras para, mediante el contraste y el cotejo, acercarnos más a la objetividad investigadora. Otros manuales que nos serían de muchísima utilidad son la obra de Peregrin, Rudimentos de bereber-rifeño, editado en Tetuán en 1944 y el Diccionario árabe-tamazight, de Mohamed Chafik, editado en Rabat en 1987.

Como indicaba en un principio, la investigación activa y unos participantes con inquietudes por la lengua y cultura tamazight son los dos mejores ingredientes capaces de garantizarnos la consecución de los objetivos que con este seminario se pretenden alcanzar.

Claves de interés para la investigación del contexto amazigh se ha de entender como una formula viable y operativa para adentrarse en la investigación de una lengua y una cultura tradicionalmente oral, que precisa ser rescatada. Este trabajo ofrece las ideas generadoras suficientes así como las claves que pueden lograr el éxito de este seminario.

La codificación metodológica de la lengua tamzight no se inicia hasta no hace mas de un siglo aproximadamente. Por la ausencia normativa de esta lengua sus hablantes jamás procedieron a su enseñanza y desarrollo. No obstante siempre la han aprendido por audición y la hablan instintivamente con absoluta naturalidad.

Se ha de resaltar la remota antigüedad de sus inicios (Prehistoria), así como su extendido espacio geográfico: Desde el Atlántico hasta las fronteras occidentales de Egipto y desde el Mediterráneo hasta Senegal, Malí, Níger y Chad. Y apreciando estos tres factores juntos (Ausencia normativa, remota antigüedad y extensión geográfica) observaremos que la lengua tamazight se ha ramificado en distintas variantes regionales, variando unas de otras: en acento y musicalidad, en cambios fonéticos y en la sustitución de vocablos por otros en los usos metonímicos y sarcásticos.

Por las características peculiares del contexto que nos ocupa, la investigación no puede circunscribirse ni realizarse exclusivamente en un gabinete o en una biblioteca. Se requiere, necesariamente, acudir para ello al espacio concreto y más amplio para esta labor. Hemos de sumergirnos en la cultura que vamos a estudiar, para tratar de comprenderla desde dentro.

De alli que las técnicas a emplear para una mejor optimización de la objetividad investigadora van desde la confecciónde mapas, notas de campo, genealogías, biografías. encuestas y cuestionarios a la fotografía y la grabación sonora y audiovisual...

El trabajo de campo ha de ser la principal fuente de recursos en el proceso de investigación. Acudir al medio geográfico propio del tamazight y los imazighen implica poder descubrir con más acierto los elementos y condicionantes que han influido sobre el hombre amazigh y sobre la configuración de su lengua. Cabe destacar que el trabajo de campo y sus aportaciones nos permitirán hacer un estudio, más enriquecedor. sobre la documentación escrita, al contar con elementos que nos facilitan el contraste y la constatación. 
Lo idóneo para abordar una empresa de estas dimensiones sería la creación de un Centro de Estudios Tamazight o por lo menos ir sentando las bases para su futura constitución. En ambos casos las funciones a desempeñar por el equipo de trabajo son, en líneas generales: recoger información, investigar y difundir la información.

Aunque a Continuación se exponen algunas ideas y sugerencias relativas a como planificar la labor investigadora del contexto amazigh, el diseño concluyente del mismo es tarea del equipo de trabajo que vaya a constituirse.

\section{Recoger información}

- Toda la que se obtenga mediante el trabajo de campo.

- Documentos procedentes de: Reuniones, conferencias, seminarios, congresos, simposios, entrevistas, etc.

- Documentación publicada: Libros, revistas, publicaciones periódicas, tesinas, tesis, estadísticas datos, informes, filmes, cintas magnetofónicas, discos, videos documentales, fotografías, imágenes, diapositivas, microfilms, folletos, guías, etc

Como elementos básicos de la investigación documental se considerarían:

- Las fuentes bibliográficas.

- Las fuentes historiográficas.

- Las biografías.

- Los diccionarios lingüísticos y toponímicos.

- Mapas topográficos.

Para poder realizar el almacenamiento de la documentación su análisis y posterior clasificación, el centro de documentación debe estar dotado de espacios suficientes y medios técnicos adecuados que garanticen su dinamización.

El centro de documentación comprendería: Una biblioteca, una sala de lectura y documentación, con terminal de ordenador, un local polivalente donde puedan realizarse trabajos de grupos así como para proyección y/o audición, y un pequeño taller de reprografía.

\section{Confeccionar relaciones de}

- Instituciones que se dedican a esta investigación.

- Asociaciones que se interesan por el fomento y desarrollo de la cultura tamazight.

- Estudiosos de la materia.

- Publicaciones periódicas.

- Otras instituciones que se dedican a investigar lenguas de similares o parecidas circunstancias y/o características que la lengua tamazight.

- Etc.

El fondo documental adquirido será en el proceso de investigación y exploración un elemento asesor y un instrumento orientativo en el trabajo de campo. 


\section{Trabajo de campo}

- Recoger mediante fotografía y vídeo el escenario y las escenas de la actividad laboral de los artesanos, ganaderos, agricultores, etc.

- Grabar los procesos de realización de estos oficios que vienen desempeñándose sin apenas alteración desde hace siglos.

- Registrar el vocabulario, los términos, la fonología, el léxico y las expresiones tanto corporales como verbales, de uso y práctica en estas artes y oficios.

- Fotografiar cada uno de los instrumentos y herramientas de uso en estas labores y realizar el registro sonoro de sus respectivos nombres y usos.

- Fotografiar y/o grabar las viviendas rústicas (exterior e interior) construidas con los elementos y métodos ancestrales.

- Rescatar y recopilar desde dentro del medio cultural amazigh y de la memoria de los mas mayores, especialmente las mujeres: La poesía tamazight antigua, el refranero, las adivinanzas y chistes, los cuentos y fábulas, las expresiones sarcásticas, burlescas y metonímicas del lenguaje por su valor descriptivo y lingüístico, y la versión que tienen sobre la historia reciente de la que han sido testigos.

- Contactar con los músicos tradicionales (imedyazen) y obtener de ellos la mayor cantidad posible de información y conocimiento sobre la música, poesía y prosa tamazight. - Localizar y/o situar con la ayuda de mapas topográficos la toponímia de la zona, estudiar su origen etimológico y los aspectos históricos que pudieran estar relacionados. - Localizar y/o situar sobre mapas topográficos lo siguiente:

- Los itinerarios comerciales, militares, culturales... de la antigüedad.

- Centros urbanos célebres desde la antigüedad.

- Mezquitas de interés histórico cultural.

- Zonas habitadas históricamente por los Churafá.

- Moravitos, cofradías sufíes, cementerios, zocos...

- Registrar los nombres de: Plantas, animales, pájaros, peces, insectos, accidentes geográficos, enfermedades y sus síntomas...

- Hacer del cuaderno de notas la memoria que registra todo cuanto vemos, oímos, pensamos o imaginamos antes, durante y después de realizar cualquier exploración. El cuaderno de notas es la chispa que alimentará nuestra imaginación y creatividad es, pues, nuestro mejor aliado.

- Es de interés gravar en vídeo algunos informativos de la TV marroquí y argelina en tamazight así como los informativos y demás programas de sus respectivas emisoras de radio.

Almacenar, analizar y clasificar la información recibida, además de permitirnos realizar las correspondientes consultas inmediatas y directas, nos va a permitir explotar con mayor y mejor rentabilidad sus contenidos en los trabajos de grupo y en el taller de lengua. La información adquirida nos será de utilidad tanto para la investigación, lingüística como para la histórico cultural. 


\section{Taller de lengua}

- Iniciar la investigación lingüistica con la vertiente del tamazight propia de la zona de Melilla y su entorno más inmediato.

- Crear un grupo de trabajo para el taller de lengua integrando en el mismo, como elemento base y fundamental, a mujeres adultas representativas de cada una de las tribus (Kábilas) que componen la provincia inmediata a Melilla.

- El objeto de estas sesiones es el registro sonoro del vocabulario amazigh así como sus aplicaciones y fonología. De igual modo se pretende rescatar el vocabulario y el lenguaje en desuso en las ciudades.

- De entre las técnicas a destacar para la consecución de estos objetivos citaremos la técnica de la imagen palabra generadora consistente en motivar y estimular a las participantes mediante la proyección o exposición de imágenes con el objeto de provocar la reaparición del vocabulario y el lenguaje contenido en al memoria. Una imagen de la vida rural y sus labores seria lo bastante sugerente para suscitar la nostalgia y la memoria de las adultas.

- Los recursos técnicos adecuados, el espacio suficiente y la metodología, previamente estudiada, contribuirán eficazmente en la dinamización de las sesiones en grupo. La grabación sonora del desarrollo y tratamiento de la lengua en su respectivo taller supone parte importante en el trabajo, tanto por motivos técnicos como documentales.

- Es importante disponer de los medios. adecuados para hacer de las grabaciones magnetofónicas, de las sesiones, un recurso de óptima calidad en sonido. El contenido de las grabaciones y la secuenciación temática del mismo habrán de ser preparados previamente en guiones para aportar la calidad deseada.

- Sería importantísimo acceder a la experiencia y a los mecanismos de estudio e investigación que se aplican en el desarrollo y fomento de la lengua vasca por ser un idioma de reciente normalización o en fase de concluirse. La reelaboración de la información obtenida relativa al vocabulario se procederá a su análisis y codificación utilizando para ello tres alfabetos: tifinagh, árabe y latín. Por razones estrictamente técnicas y académicas los registros escritos de la fonología tamazight deben de realizarse, simultáneamente, en estos tres alfabetos. La exclusión de cualquiera de ellos supondría una mutilación en el mecanismo de la investigación.

En la fase de estudio y análisis del vocabulario y sus aplicaciones se tendrá en cuenta entre otras apreciaciones lo siguiente:

- Discriminar el vocabulario amazigh original de otros prestados.

- Constatar si el medio geográfico y subsistencial han influido en la generación de lexicografías distintas entre las variantes del tamazight.

- Estudiar la estructura lingüística base del tamazight y averiguar si las distintas variantes de la misma se rigen gramaticalmente por idénticas normas constructivas y estructurales. - Confeccionar una relación del vocabulario de uso común entre las distintas variantes del tamazight y estudiar los contrastes fonológicos más apreciables.

- Aplicar sobre la información obtenida para su análisis, clasificación, catalogación y archivo las técnicas de investigación más acordes, así como la metodología que sobre 
la marcha el equipo de investigación tendría que idear y planificar (Creatividad propia en la búsqueda y tratamiento de la información).

- Idear un mecanismo de ficheros y archivos integrales capaces de satisfacer al máximo las necesidades de los interesados en la materia $(E$ : Combinar el fichero lingüístico con cintas magnetofónicas + vídeo + fótografía + orientaciones hacia documentación escrita).

- Conforme se vaya avanzando en la exploración de la zona inmediata a Melilla, el radio de acción en torno a la misma se irá ampliando progresivamente hasta cubrir el máximo que nos permitan nuestros medios y recursos. Una vez cubiertos los objetivos trazados relativos a la entrada y reelaboración de los distintos soportes documentales entraríamos en la fase consistente en servir y distribuir la información tratada. La salida de la información podría adoptar las siguientes formas entre otras:

- Poner la información archivada y clasificada a disposición de los estudiosos.

- Asesorar a los medios de comunicación.

- Editar monográficos.

- Publicar diccionarios.

- Elaborar traducciones (Cuentos, fábulas, refraneros, etc.)

- Memorias, cuadernos, publicaciones periódicas...

- Organizar cursos, seminarios, simposios.

- Servicios de reprografía.

- Materiales didácticos.

- Etc.

La actividad externa en la distribución de la información nos permitirá, indudablemente, evaluar nuestra eficacia y advertir las posibles lagunas en nuestros mecanismos de funcionamiento.

Como se expuso en un principio la intención de este resumido trabajo no es otra que la de mostrar algunas claves de interés para la investigación del contexto amazigh. Abordar la cuestión desde sus distintas y complejas perspectivas es un trabajo de equipo cualificado, organizado y coordinado. 
TAMEDRUT TIMZIGIT N YIRES D TADELSA TAMAZIĠT SEMINARIO PERMANENTE DE LENGUA Y CULTURA TAMAZIGHT

El alfabeto rifeño y su trascripción en caracteres árabes y latinos

\begin{tabular}{|c|c|c|c|c|c|c|}
\hline \multicolumn{2}{|c|}{ Tifinagh } & \multirow[t]{2}{*}{ Nombre } & \multirow{2}{*}{$\begin{array}{c}\text { Alfabeto } \\
\text { árabe }\end{array}$} & \multirow{2}{*}{$\begin{array}{l}\text { Transliteración } \\
\text { estándar }\end{array}$} & \multirow{2}{*}{$\begin{array}{c}\text { Transliteración } \\
\text { utilizada }\end{array}$} & \multirow[t]{2}{*}{ Equivalencia } \\
\hline May. & Min. & & & & & \\
\hline$\dot{8}$ & $\cdot$ & $\mathbf{a}$ & $i$ & $a$ & $\mathbf{a}$ & a anterior con tendencia a e \\
\hline$\Phi$ & $\Phi$ & ba & 4 & b & $\mathbf{b}$ & b \\
\hline+ & + & ta & $ت$ & $t$ & $\mathbf{t}$ & $t$ \\
\hline$x$ & $x$ & ta & $\Delta$ & $t$ & 1 & $\mathbf{z}$ \\
\hline 8 & $\vdots$ & ilem & 1 & $\mathbf{e}$ & $\mathbf{e}$ & e neutra ó débil \\
\hline $\mathbf{I}$ & $I$ & ja & $\varepsilon$ & $\mathbf{j}$ & $\mathbf{j}$ & $\mathrm{j}$ inglesa \\
\hline $\mathbf{z}$ & $\mathbf{z}$ & yedj & $\varepsilon^{d}$ & $\check{\mathbf{g}}$ & dj & dfendiboutt dicoconfiancesa \\
\hline$\Lambda$ & 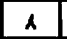 & hạ & $\tau$ & $\underline{\mathbf{b}}$ & $\underline{\mathbf{h}}$ & hespirante farthgea sorda \\
\hline$x$ & $x$ & $\mathbf{x a}$ & $E$ & $x$ & $\bar{x}$ & 1 \\
\hline$\Lambda$ & $\Lambda$ & da & 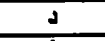 & d & d & d \\
\hline $\mathrm{V}$ & $v$ & da & $\dot{j}$ & d & $\underline{\mathbf{d}}$ & Thinglesenthe \\
\hline$\varepsilon$ & $\varepsilon$ & $\mathbf{i}$ & $!$ & $\mathbf{i}$ & $\mathbf{i}$ & $\mathbf{i}$ \\
\hline 0 & 오 & $\mathbf{r a}$ & 2 & $\mathbf{r}$ & $\mathbf{r}$ & $\mathbf{r}$ \\
\hline $\mathrm{Q}$ & 오 & r̦ą & $\jmath$. & $\underline{r}$ & $\underline{r}$ & Fsulave omitible \\
\hline ૉ & $\underline{x}$ & $\mathbf{z a}$ & $j$ & $\mathbf{z}$ & $\mathbf{z}$ & zfancesa \\
\hline $\mathbf{x}$ & $\mathbf{x}$ & zar & $j$ & $\mathbf{z}$ & $\underline{z}$ & zframcesa nas \\
\hline$\odot$ & 0 & Sa & س & s & $\mathbf{s}$ & s \\
\hline$\sigma$ & 0 & sar & w & $\underline{s}$ & $\$$ & syelarizada \\
\hline $\mathrm{C}$ & c & ca & ث & c & c & shing osq \\
\hline $\mathbf{c}$ & $\mathbf{c}$ & tca & تصن & $\check{\mathbf{c}}$ & ç & ch \\
\hline $\mathbf{E}$ & $\mathbf{E}$ & dad & ض & d & $d$ & indergada \\
\hline$\exists$ & $\exists$ & da & $\mathbf{B}$ & $\underline{d}$ & $\underline{d}$ & dolarizads \\
\hline$E$ & $E$ & ta & $b$ & $t$ & $!$ & Tht hvelarigog a \\
\hline ค & ค & $\mathbf{c i l}$ & $\varepsilon$ & $\varepsilon$ & $1<$ & espiranteffringea sonora \\
\hline$\Psi$ & $\psi$ & $\dot{\mathbf{g} a}$ & $\dot{\varepsilon}$ & $\gamma$ & $\mathbf{g}$ & Ipdinga \\
\hline$\underline{H}$ & I & $\mathrm{fa}$ & i & $\mathbf{f}$ & $f$ & $\mathbf{f}$ \\
\hline Z & $\mathbf{z}$ & qil & 3 & 9 & q & Kưular $\quad$ at \\
\hline $\mathbf{R}$ & $\mathbf{n}$ & ka! & $s$ & $\mathbf{k}$ & k & $\mathbf{k}$ \\
\hline $\bar{Z}$ & $x$ & ga & $s$ & $\mathbf{g}$ & $\mathbf{g}$ & g castellana en galgo \\
\hline$\|$ & 1 & la & J & 1 & $\mathbf{I}$ & 1 \\
\hline[ & ᄃ & ma & s & $\mathbf{m}$ & $\mathbf{m}$ & $\mathbf{m}$ \\
\hline 1 & 1 & na & $\dot{0}$ & $\mathbf{n}$ & $\mathbf{n}$ & n \\
\hline$:$ & $:$ & $\mathbf{u}$ & 1 & $\mathbf{u}$ & $\mathbf{u}$ & $\mathbf{u}$ \\
\hline$\phi$ & $\Phi$ & ha & $\Delta$ & h & $\mathbf{h}$ & 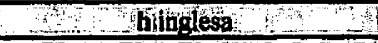 \\
\hline U & U & wa & 9 & $\mathbf{w}$ & $\mathbf{w}$ & Ar semiconsoinante \\
\hline$\Pi$ & $n$ & ya & 9 & $\mathbf{y}$ & $\mathbf{y}$ & Heriticonsonante \\
\hline
\end{tabular}




\section{Pronombres demostrativos}

Sustituyen al nombre e indican su relación con las personas gramaticales y el espacio y el tiempo.

"Ejemplos:
- Wa d uma.
- Tin d tagarbuzt $n$ mmi.
- Win d amedduker $n$ Farid.
- Aytma dina.
- Ta dxaci.
- Tisermadin inu do tinin.

\begin{tabular}{|c|c|c|c|c|c|}
\hline \multirow{3}{*}{$\begin{array}{l}\text { - Proximidad en el } \\
\text { espacio al hablante }\end{array}$} & \multicolumn{3}{|c|}{ Singular } & \multicolumn{2}{|c|}{ Plural } \\
\hline & Masculino & Femenino & Neutro & Masculino & Femenino \\
\hline & $\begin{array}{l}\text { Wa } \\
\text { Éste }\end{array}$ & $\begin{array}{c}\text { Ta } \\
\text { Ésta }\end{array}$ & $\begin{array}{c}\text { Ay-a } \\
\text { Esto }\end{array}$ & $\begin{array}{l}\text { Ina } \\
\text { Éstos }\end{array}$ & $\begin{array}{l}\text { Tina } \\
\text { Estas }\end{array}$ \\
\hline $\begin{array}{l}\text { - Cierta proximidad en } \\
\text { el espacio al hablante. }\end{array}$ & $\begin{array}{l}\text { Win } \\
\text { Ese }\end{array}$ & $\begin{array}{l}\text { Tin } \\
\text { Ésa }\end{array}$ & $\begin{array}{c}\text { Ay-in } \\
\text { Eso }\end{array}$ & $\begin{array}{l}\text { Inin } \\
\text { Esos }\end{array}$ & $\begin{array}{l}\text { Tinin } \\
\text { Esas }\end{array}$ \\
\hline $\begin{array}{l}\text { - Lejanía en el espacio } \\
\text { y tiempo al hablante y } \\
\text { oyente. }\end{array}$ & $\begin{array}{l}\text { Wenni } \\
\text { Aquel }\end{array}$ & $\begin{array}{l}\text { Tenni } \\
\text { Aquellla }\end{array}$ & $\begin{array}{l}\text { Ay-nni } \\
\text { Aquello }\end{array}$ & $\begin{array}{l}\text { Inni } \\
\text { Aquéllos }\end{array}$ & $\begin{array}{c}\text { Tinni } \\
\text { Aquéllas }\end{array}$ \\
\hline
\end{tabular}

Pronombres posesivos

Siven para indicar que lo nombrado por el sustantivo al que hacen referencia pertenece a la primera, segunda o tercera persona del coloquio. El pronombre posesivo depende, en su género y número, del poseedor y no del objeto o la cosa poseída.

"Ejemplos:

$\begin{array}{ll}\text { - Aqrab-a inu. } & \text { - Yis-nni abarcan nsen. } \\ \text { - Tisira-ya nnec. } & \text { - Ahenjar-in ncent. } \\ \text { - Taddart-in tameqrant nsen. } & \text { - Thenjirin-in ncent. }\end{array}$

\begin{tabular}{|c|c|c|c|c|c|}
\hline$I^{\circ P}$ & ona & & & & \\
\hline & nứn & Masculino & Femenino & & \\
\hline & $\ln u$ & Nnec & Nnem & & \\
\hline & $\mathrm{Hio} / \mathrm{s}-\mathrm{Mh} / \mathrm{s}$ & & & Suyo & $y y a / s$ \\
\hline & & & & Masculino & Femenino \\
\hline PI. & Nneg & Nwem & Ncent & Nsen & Nsent \\
\hline Nu & $\mathrm{ro} / \mathrm{s}-\mathrm{Nu}$ estra/ & Vuest & stra/s & Suy & suya/s \\
\hline
\end{tabular}


Jahfar Hassan Yahia

\section{Determinantes demostrativos sufijos}

Se posponen al nombre y lo determinan, contribuyendo a simplificar la oración, en la que interviene el pronombre demostrativo, haciéndola más corta sin alterar ni en lo más mínimo el mensaje, siempre y cuanda la conversión sea admisible.

*Ejemplos:

$\begin{array}{ll}\text { - Wa d ahenjar inu. } & \text { - Ahenjar-a inu. } \\ \text { - Ta d taddart n uma. } & \text { - Taddart-a n uma. } \\ \text { - Win d aryaz d azirar. } & \text { - Anyaz-in d azirar. }\end{array}$

\begin{tabular}{|c|c|c|c|}
\hline \multirow{3}{*}{$\begin{array}{l}\text { - Proximidad en el } \\
\text { espacio al hablante. }\end{array}$} & & \multicolumn{2}{|c|}{ Para ambos géneros y números } \\
\hline & Sustantivo acabado en consonante & $-\mathbf{a}$ & \multirow{2}{*}{ Éste - Éstos, ésta/s } \\
\hline & Sustantivo acabado en vocal & -ya & \\
\hline \multirow{3}{*}{$\begin{array}{l}\text { - Cierta proximidad en } \\
\text { el espacio al hablante } \\
\text { - Lejanía en el espacio y } \\
\text { tiempo al hablante y oyente. }\end{array}$} & $\begin{array}{l}\text { Sustantivo acabado en consonante } \\
\text { Sustantivo acabado en vocal }\end{array}$ & $\begin{array}{l}- \text { in } \\
- \text { yin }\end{array}$ & Ése - Éso, Ésa/s \\
\hline & Sustantivo acabado en consonante & $-n n i$ & \multirow{2}{*}{ Aquél - Aquéllos, Aquélla/s } \\
\hline & Sustantivo acabado en vocal & $-n n i$ & \\
\hline
\end{tabular}

Los géneros y números del nombre

Los nombres en la lengua tamazight no tienen artículo propiamente dicho: mas su género y número gramaticales se conocen fácilmente por el prefijo y/o sufjo que entran en la formación de los mismos y que presentan distintas formas en su conformidad con el género y número de los nombres. El cuadro siguiente nos muestra cómo se constituye regularmente el género y número de los nombres mediante éstos afijos nominales con sus diferentes formas gramaticales.

\begin{tabular}{|l|l|l|}
\cline { 2 - 3 } \multicolumn{1}{c|}{} & Singular & Plural \\
\hline Masculino & ahenjar & ihenjaren \\
\hline Femenino & tahnjart & tihenjarin \\
\hline Masculino & azirar & iziraren \\
Femenino & tazirart & tizirarin \\
Masculino & abarcan & ibarcanen \\
\hline Femenino & tabarcant & tibarcanin \\
\hline Masculino & afunas & ifunasen \\
\hline Femenino & tafunast & tifunasin \\
\hline
\end{tabular}




\section{Los determinantes sufijos en los parentescos}

Los nombres de parentesco como baba, yemma, mmi, yedji, uma, wucma, jeddi, henna, <emmi, <enti, xari, xaci etc. (respectivamente mi padre, mi madre, mi hijo, mi hija, mi hermano, mi hermana, mi abuelo, mi abuela, mi tío paterno, mi tía paterna, mi tío matemo, mi tía materna) son automáticamente atribuidos a un poseedor primera persona del singular. No obstante éstos determinantes sufijos denotan más bien referencia que posesión.

El sufijo de la primera persona singular es a con unos nombres; y con otros i; y en el plural toman, éstos sufijos. una ț fricativa delante de sí.

Contrastad éstos afijos con los de los pronombres posesivos de la página 99.

\begin{tabular}{|c|c|c|c|c|c|c|}
\hline \multirow{4}{*}{ 莺 } & \multicolumn{2}{|c|}{ Determinantes suffjos } & \multicolumn{4}{|c|}{ Nombres de parentescos en singular } \\
\hline & $1^{\mathrm{a}}$ Pers. común & $a-i$ & Baba & Yemma & Mmi & Yedji \\
\hline & $2^{\mathrm{a}}$ Pers. masculina & $c / k$ & $\mathrm{abac} / \mathrm{k}$ & Yemr & $\mathrm{Mn}$ & Yedjic/k \\
\hline & $2^{\mathrm{a}}$ Pers. femenina & $\mathbf{m}$ & Babam & Yen & $\mathrm{M}$ & $\mathrm{m}$ \\
\hline & $3^{\mathrm{a}}$ Pers. común & s & Babas & Yen & $M$ & $Y_{e d}$ \\
\hline \multirow{5}{*}{ 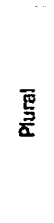 } & $1^{\mathrm{B}}$ Pers. común & $t$ neg & Babatneg & Yemma & $\mathrm{Mn}$ & Yed \\
\hline & $2^{\mathrm{a}}$ Pers. masculina & twem & Babatwem & natwem & $\mathrm{Mn}$ & Yedjitwem \\
\hline & $2^{a}$ Pers. femenina & tcent/kent & $\begin{array}{l}\text { Babat cent } \\
t \text { kent }\end{array}$ & $\begin{array}{l}\text { Yemmatcent } \\
\text { tkent }\end{array}$ & $\begin{array}{l}\text { Mmitcent/ } \\
t \text { kent }\end{array}$ & $\begin{array}{l}\text { Yedjitcent } \\
\text { tkent }\end{array}$ \\
\hline & $3^{\mathrm{a}} \mathrm{Pe}$ & $t$ se & Babatsen & Yem & & Yedjitsen \\
\hline & $3^{a}$ Pers. $f$ & $t$ sent & Babatsent & Yemmatsent & Mmit sent & Yedjitsent \\
\hline
\end{tabular}

Los pronombres personales nominativos

Los pronombres personales nominativos son las palabras utilizadas para designar las personas del discurso, indicando el rol de cada individuo.

- La $1^{\mathrm{a}}$ persona indica al hablante.

- La $2^{\mathrm{a}}$ persona indica al oyente.

- La $3^{a}$ persona hace referencia a los demás, los que están fuera de la conversación.

\begin{tabular}{|c|c|c|c|c|c|c|c|c|c|}
\hline \multicolumn{2}{|c|}{ 1- PERSONA } & \multicolumn{4}{|c|}{$2^{\circ}$ PERSONA } & \multicolumn{4}{|c|}{ 30 PERSONA } \\
\hline Singula & ar Plural & \multicolumn{2}{|c|}{ Singular } & \multicolumn{2}{|c|}{ Plural } & Sing & & \multicolumn{2}{|c|}{ Plural } \\
\hline \multicolumn{2}{|c|}{ Común } & Mascutino & Femenino & Masculino & Femenino & Mascultino & Femenino & Masculino & Fernonino \\
\hline $\mathrm{Nec}$ & Neccin & Cek & Cem & Kenniw & Kennint & Netta & Nettat & Nitnin & Nițint / Nitenti \\
\hline Yo & $\begin{array}{l}\text { Nosotros, } \\
\text { Nosotras }\end{array}$ & $T u$ & $T u$ & Vosotros & Vosotras & $\dot{E}$ & Ella & Ellos & Ellas \\
\hline
\end{tabular}


Para mayor información sobre los determinantes sufijos y los nombres de parentesco cotejad la presente con la página 101.

\section{A}

Nombres de parentesco que precisan de los determinantes posesivos atjos

\begin{tabular}{|c|c|c|c|c|}
\hline \multicolumn{2}{|c|}{ Masculino } & \multicolumn{2}{|c|}{ Femenino } & \multirow[t]{2}{*}{ Equivalente en castellano } \\
\hline Singular & Plural & Singular & Plural & \\
\hline baba & Iwalidin, ibabaten & yemma & tiyimmaţin & Padre, madre \\
\hline mmi & Arraw, tarrawt & yedji & yessi & Hijo, hija-s \\
\hline uma & ayțma & wucma & yesma & Hermano/a-s \\
\hline$<$ emmi & $<$ mumi & <enti & <wanti & Tio/a-s paterno/a-s \\
\hline xari & xwari & xatci & xwatci & Tio/a-s matemo/a-s \\
\hline jeddi & rejdud & ḥenna & thẹennatin & Abuelo, abuela \\
\hline & & lalla & tillallațin & Suegra del marido y de la mujer \\
\hline
\end{tabular}

\section{B}

Nombres de parentesco ajenos a los determinantes posesivos afijos

\begin{tabular}{|c|c|c|c|c|}
\hline \multicolumn{2}{|c|}{ Masculino } & \multicolumn{2}{|c|}{ Femenino } & \multirow{2}{*}{ Equivalente en cestellano } \\
\hline Singutar & Plurat & Singular & Plural & \\
\hline Baba d yemma & Iwalidin & tayemmat & tiyimmațin & Padres (del árabe), madre-s \\
\hline jeddi & rejdud & tahenennat & tị̣ennaţin & Abuelos (del árabe), abuela-s \\
\hline mmi & arraw / tarrawt & yedji & yessi & Hijos, prole, descendencia \\
\hline ayyaw & ayyawen & tayyawt & tayyawin & Nieto/a-s \\
\hline asrif & isrifen & tasrift & tisrifin & $\begin{array}{l}\text { Cuñado-s: marido de la hermana de una } \\
\text { mujer, los maridos de dos hermanas son } \\
\text { isrifen. Cuñada-s: hermana de la esposa }\end{array}$ \\
\hline aḍgwar & iḍuwran & taḍugwatc & tiḍuwrin & Suegro/a-s del esposo. Parientes poltticos \\
\hline amġar & imugar & tamǵart & timugar & Suegro/a-s de la esposa \\
\hline & & tanuṭ & tinuḍin & $\begin{array}{l}\text { Concuñada-s: esposa del hermano } \\
\text { del marido }\end{array}$ \\
\hline arbib & arbiben & tarbibt & tarbibin & Hijastro/a-s \\
\hline arews & irewsan & tarewst & tirewsin & $\begin{array}{l}\text { Cuñado-s: hermano del marido. } \\
\text { Cuñada-s: hermana del marido }\end{array}$ \\
\hline acniw & acniwen & tacna & tacniwin & Mellizo/a-s, coesposa-s \\
\hline amenzu & imenza & tamenzut & timenza & Primogénito/a-s \\
\hline amazuz & imazuzen & tamazuzt & timazuzin & Benjamin/a-s \\
\hline
\end{tabular}




\section{El verbo $\angle N A=S E R$ y su uso restringido}

Las letras preformativas y aformativas (Prefijos y sufijos) de la conjugación.

El pronombre interrogativo $M I N=$ QUÉ. (Qué o quién si se usa con el verbo <NA).

Respecto al verbo <NA nos interesa del mismo su pretérito, que es el tiempo que más se usa en su caso, por tratarse de un verbo de estado cuya utilización se restringe a expresiones muy concretas pero a la vez muy usuales $y$ frecuentes. Ver ficha verbal.

El pretérito: el pretérito expresa el pasado en el caso de un verbo de acción: yufa (él encontró). pero también el presente en el caso de un verbo de estado: yewsar [él es/era viejo].

La radical del verbo: la radical o raíz del verbo se obtiene del imperativo de la segunda persona singular: ARI (escribe) $=$ ESCRIBIR, la forma del infinitivo del verbo es inexistente en la lengua tamazight al igual que en el resto de las lenguas camitosemíticas.

\begin{tabular}{|lll|}
\hline Nec & e<nig & $------\dot{g}$ \\
Cek & te<nid & $t-----d$ \\
Cem & te<nid & $t-----d$ \\
Netta & ye<na & $i / y----$ \\
Nettat & te<na & $t------$ \\
Neccin & ne<na & $n------$ \\
Kenniw & te<nam & $t-----m$ \\
Kennint & te<nant & $t-----n t$ \\
Nițin & e<nan & $------n$ \\
Nițenti & e<nant & $-----n t$ \\
\hline
\end{tabular}

La conjugación del verbo: la conjugación del verbo en la lengua tamazight se realiza mediante los prefjos y/o sufjios del esquema anterior que indican la variación del género y número de las personas en la conjugación. 
La particula conjugable "aqqa" (he + pronombre personal acusativo) en uso con el pronombre personal acusativo [c.d] equivalente al presente de indicativo del verbo estar. $Y$ la partícula pretérita "tuga" en uso también con el pronombre personal acusativo (c.d) equivalente al pretérito imperfecto o indefinido de los verbos sery estar

\begin{tabular}{|c|c|c|c|c|c|}
\hline & $\begin{array}{l}\text { Forma inabreviable } \\
\text { excepto en } \\
\text { "Cek" aqqa-c }\end{array}$ & $\begin{array}{l}\text { Equivalente } \\
\text { en casteilano }\end{array}$ & $\begin{array}{l}\text { Forma inabreviable } \\
\text { excepto en } \\
\text { "Cok" tuga-c }\end{array}$ & Equivalente en castellano & \\
\hline $\mathrm{Nec}$ & $a q q-a i$ & estoy & tuǵ-ai & estaba/estuve & era/fui \\
\hline Cek & aqqa-cek & estás & tugia-cek & estabas/estuviste & eras/fuiste \\
\hline Cem & aqqa-cem & estás & tuġa-cem & estabas/estuviste & eras/fuiste \\
\hline Netta & aqqa-t & está & tuga-t & estaba/estuvo & era/fue \\
\hline Nettat & aqqa-t & está & tuġa-t & estaba/estuvo & era/fue \\
\hline Neccin & aqqa-neg & estamos & tuġa-neg் & estabamos/estuvimos & éramos/fuimos \\
\hline Kenniw & aqqa-kenniw & estáis & tuġa-kenniw & estabais/estuvisteis & erais/fuisteis \\
\hline Kennint & aqqa-kennint & estáis & tuga-kennint & estabais/esturisteis & erais/fuisteis \\
\hline Nitnin & aqqa-țen & están & tugia-țen & estaban/estuvieron & eran/fueron \\
\hline Nitenti & aqqa-tent & están & tugia-țent & estaban/estuvieron & eran/fueron \\
\hline
\end{tabular}

Ejemplos:

- Karim aqqa-t di rbit nnes.

- Xari d mmis aqqa-ten akid-i.

- Nhar-a aqq-ai we;d-i.

- ¿Mermi-kenniw tuga di Figig?

* Tug a-neg din simana i ye<dun.

- ¿Mani-cek/m tuga gar uzien nhar?

* Tug-ai di rxedmet, am rebda.

- Farid tugia-t $\mathbf{d}$ anejjar di Fas.

- Tinin tuga-tent d disermadin inu.
- Karim está en su habitación.

- Mi tó y su hijo están conmigo.

- Hoy estoy solo.

- ¿Cuándo estuvisteis en Figuig?

* Estubimos alli la semana pasada.

- ¿Dónde estuviste al medio día?

- Estuve en el trabajo, como siempre.

- Farid era carpintero en Fés.

- Esas eran mis maestras. 
Pronombres personales en función de complemento indirecto

\begin{tabular}{|c|c|c|c|c|c|}
\hline & & & Independientes & Sufijos & \\
\hline \multirow{2}{*}{ 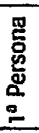 } & Singular & Común & dai & -ai & me \\
\hline & Plural & Común & daneǵ & -aneǵ & nos \\
\hline \multirow{4}{*}{ 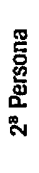 } & \multirow{2}{*}{ Singular } & Mascultino & dac & $-a c$ & te \\
\hline & & Femenino & dam & -am & te \\
\hline & \multirow{2}{*}{ Plural } & Masculino & dawem & -awem & OS \\
\hline & & Femenino & dacent/dakent & -acent/-akent & os \\
\hline \multirow{3}{*}{ 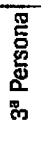 } & Singular & Común & das & -as & le \\
\hline & Plural & Masculino & dasen & -asen & les \\
\hline & & Femenino & dasent & -asent & Tes \\
\hline
\end{tabular}

Ejemplos:

- ¿Min das tucid i Racid?

* Uciǵ-as arrự nnes.

- ¿Tucid̄-as arruợ nnes wahan?

* Lla, war das ucig bu arruọ nnes wahan. uciǵ-as ura d tahezzant inu.
- ¿Qué le diste a Rachid?

* Le di su ropa.

- ¿Le diste su ropa solamente?

* No, no le dísu ropa solamente, le di tambien mi cinturón. 
Jahfar Hassen Yahia

Pronombres personales en función de complemento directo

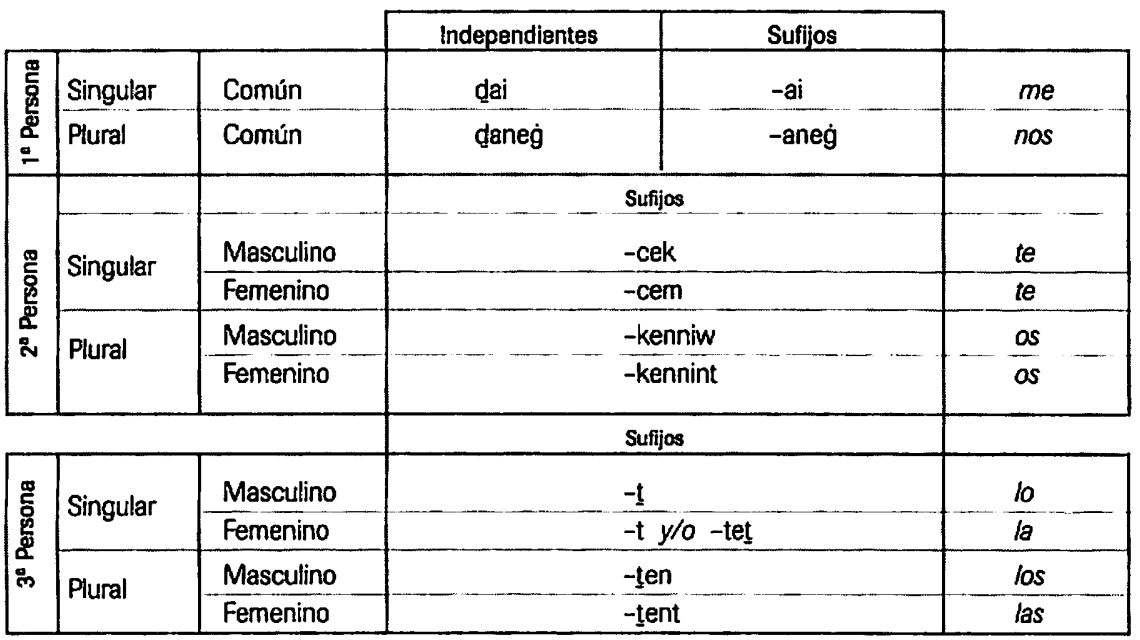

\section{Ejemplos:}

- ¿Mamec texsed atay?

* exseg̀-t d miziọ.

- ¿Cómo quieres el té?

- ¿Texsed-t mbra ssekkwar?

* Lo quiero dulce.

- ¿Lo quieres sin azúcar?

- La, war-t exseg bu mbra ssekkwar, exseǵ-t d miziḍ.

" No, no lo quiero sin azúcar, lo quiero dulce. 
Las preposiciones conjugables "Gar" y "Di" equivalentes al presente de indicativo del verbo Tener

\begin{tabular}{|c|c|c|c|c|}
\hline & \multicolumn{2}{|c|}{ "Tener" en la acepción de poseer } & \multirow{2}{*}{$\begin{array}{l}\text { tener" en la acepción de contener, padecer } \\
\text { No odmite uso abroviado }\end{array}$} & \multirow[t]{2}{*}{ Equivalente en Castellano } \\
\hline & Fonologicamente & Fonélicamente & & \\
\hline $\mathrm{Nec}$ & gar-i & $\dot{g} a r-i$ & day-i & tengo \\
\hline Cek & gar- $k / c$ & $\dot{g} a-k / c$ & day-k/c & tienes \\
\hline Cem & gar $-m$ & $\dot{g} a-m$ & day-m & tienes \\
\hline Netta & gar-s & ga-s & day-s & tiene \\
\hline Nettat & ǵar-s & ġa-s & day-s & tiene \\
\hline Neccin & ġar-neǵ & ġa-neg் & day-neg & tenemos \\
\hline Kenniw & gar-wem & ga-wem & day-wem & tenéis \\
\hline Kennint & gar-kent/cent & g̀a-kent/cent & day-kent/cent & tenéis \\
\hline Nițnin & gar-sen & ġa-sen & day-sen & tienen \\
\hline Nițenti & gar-sent & ga-sent & day-sent & tienen \\
\hline
\end{tabular}

Ejemplos:

- ¿Mechar $n$ refrus gar- $\mathrm{k} / \mathrm{m}$ ? - ¿ ¿Cuánto dinero tienes?

- Gar-i țratin $n$ eurut. $\quad$ "Tengo teinta euros.

- ¿ं்ar-k/m ay-a wahan? - ¿Tienes esto solamente?

*Wah, gar-i ay-a wahan. *Si, tengo esto solamente.

- ¿Min day-wem? - ¿Qué teneis?

* Day-neg timessi. *Tenemos fiebre.

- ¿Zi mermi day-wem timessi? - ¿Desde cuándo tenéis fiebre?

* Day-neg timessi zeg iộnnad. * * Tenemos fiebre desde ayer. 
El Imperativo Perfecto del verbo UC: DAR en uso con los Pronombres Personales en función de Complemento Directo $\left(1^{\mathrm{a}}\right.$ y $3^{\mathrm{a}}$ persona]

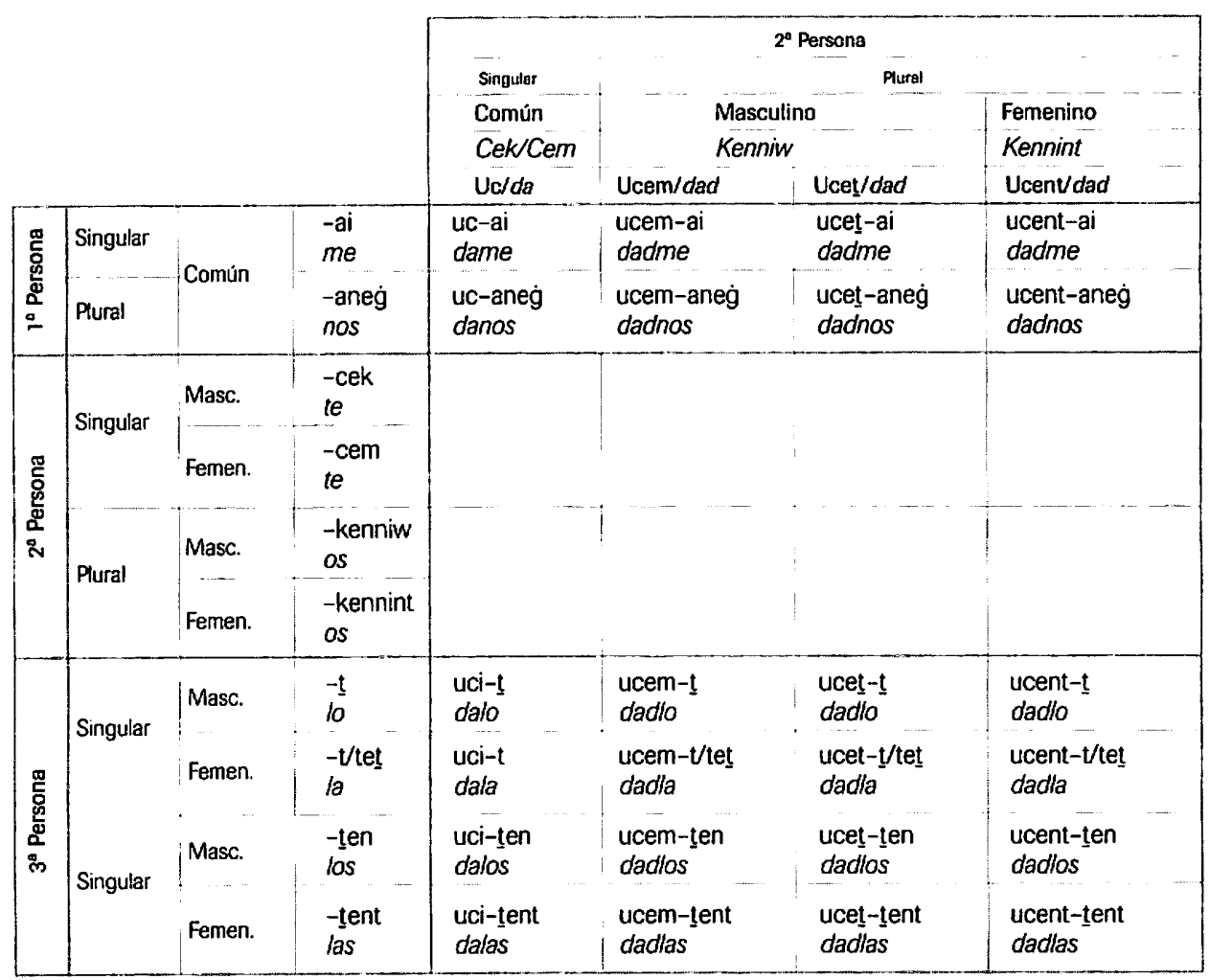

Ejemplos:

- ¿ Mermi dac ga uceg rektub ? - ¿Cuándo te doy los libros?

* Uc-ai-ten tiwucca. * Dámelos mañana.

- ¿A dac-ten uceg tiwucca? - ¿Te los doy mañana?

*Wah, uc-ai-ten tiwucca. * * Si, dámelos mañana. 
El Imperativo Perfecto del verbo UC: DAR en uso con los Pronombres Personales en función de Complemento Indirecto $\left(1^{\mathrm{a}}\right.$ y $2^{\mathrm{a}}$ persona). $Y$ en uso con el Sintagma "I IXF" y los

Pronombres Posesivos ( $2^{\mathrm{a}}$ persona)

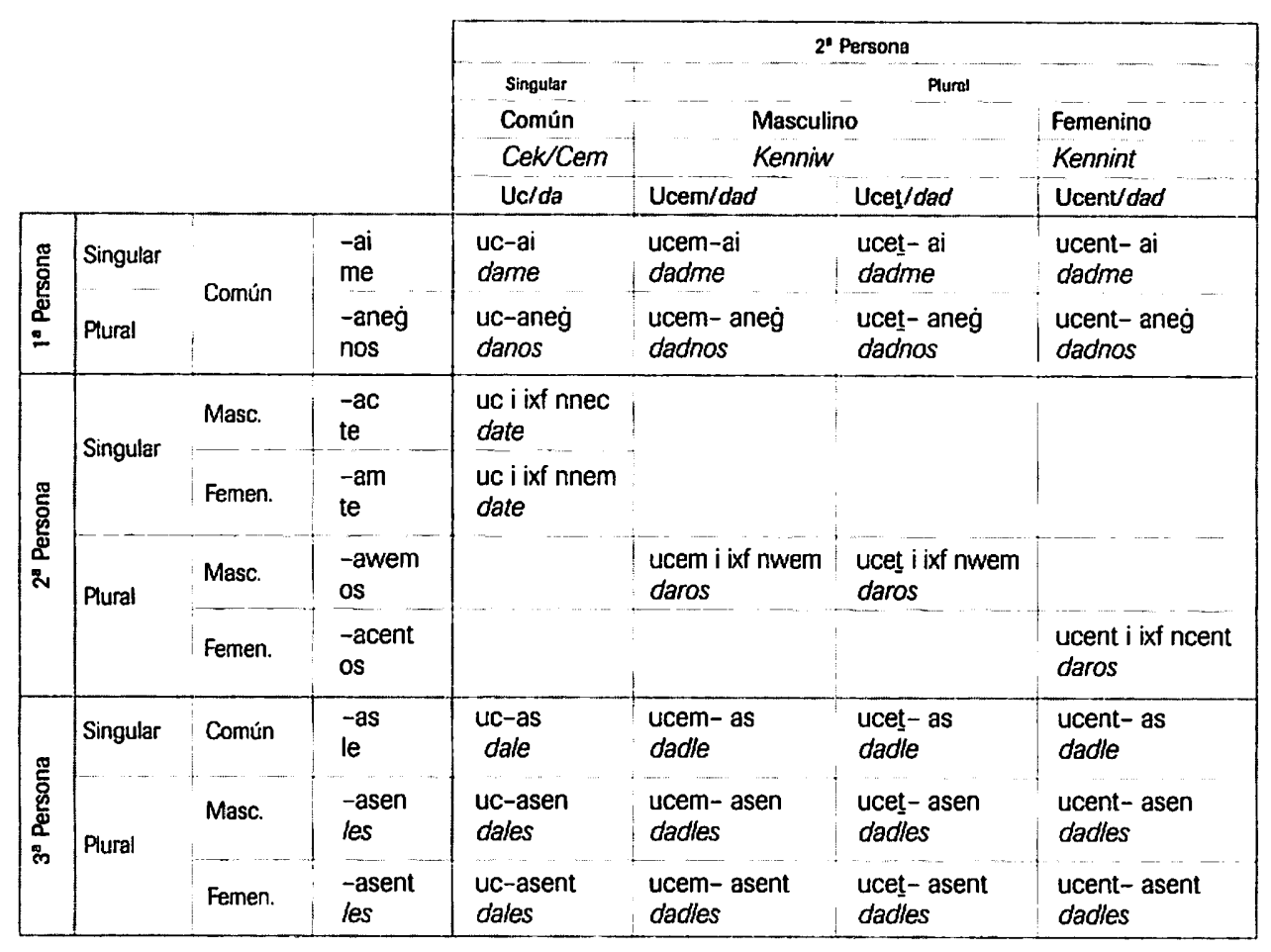

Ejemplos:

- ¿l umi ga nuc taqmijat-a? - ¿A quién le damos esta camisa?

* Ucem-as-t i Numidia. - Dádsela a Numidia.

- ¿A das-t nuc i Numidia? - ¿Se la damos a Numidia?

* Wah, ucem-as-t i nettat. *Si, dádsela a ella. 
Preposiciones

\begin{tabular}{|l|l|}
\hline Castellano & Tamazight \\
\hline A & Gar \\
Ante & Qibar \\
Bajo & Sadu \\
Con & Ak (compañfa) \\
Con & S (instrumentalización) \\
Contra & Mgal, Negguw, Mnid \\
De & Zi (procedencia) \\
De & $\mathrm{N}$ (posesión) \\
Desde & Zi \\
En & Di \\
Entre & Jar \\
Hacia & Gar \\
Hasta & Ar \\
Para & I (afecta al nombre) \\
Para & Huma (afecta al verbo) \\
Por & X \\
Según & <laḥsab \\
Sin & Mbra \\
Sobre & X \\
Trás & Awarni \\
\hline
\end{tabular}


Conjunciones

\begin{tabular}{|l|l|}
\hline Castellano & Tamazight \\
\hline A fin de que & Huma + futuro \\
A que & Yak \\
Antes & Qber \\
Antes que & Qber i \\
Aunque & Waxxa \\
Aunque & Waxxa \\
Como & Am, cnaw, am cnaw \\
Como cuando & Am cnaw xemmi \\
Como sea & Mamec ma \\
Con lo que sea & S minzi ma \\
Con que & Zixentan \\
Con quién sea & Wik ma \\
Cuando & Mermi, xemmi, ami \\
Cuando sea & Mermi ma \\
Dado que & Ami \\
Desde cuando & Zeg wami \\
Desde que & Zeg wami \\
Donde sea & Mani ma \\
Entonces & Rexdenni, uca \\
Hasta que & Ar ami \\
Igual que & Am cna \\
Luego & Rexdenni \\
Mas & Maca \\
Más & Ktar \\
Ni & Ni, ra \\
O, u & Niǵ \\
Para que & Huma + futuro \\
Pero & Maca \\
Pero & Maca \\
Por eso & X uy-nni \\
Por esto & X uy-a \\
Porque & Minzi \\
\hline & \\
\hline
\end{tabular}


Interjecciones

\begin{tabular}{|l|l|}
\hline Castellano & Tamazight \\
\hline iAh! & IAh! \\
iAnda! & iZid! \\
iAy que dolor! & iAḥ̣ i rehriq! \\
iAy! & iAḥ̣! \\
iHola! & ilwa! \\
iMi madre! & IA yemma inu! \\
iOh! & iA! \\
IOjalá! & iMaবlik! \\
iSocorro! & iAyyawa! \\
\hline
\end{tabular}

Adverbios de lugar

\begin{tabular}{|l|l|}
\hline Castellano & Tamazight \\
\hline A donde & Ar mani \\
Abajo & Swadday \\
Acá. más acá & Awrud \\
Adentro & Gar daxer \\
Afuera & Gar barra \\
Ahí & Din \\
Allá & Dinni \\
Allá, más allá & Agirin \\
Alli & Dihan \\
Aquí & Da, danit-a, danit-in \\
Arriba & sennaj \\
Bajo & Sadu \\
De ahi/allí & Ssihan \\
De allá & ssenni \\
De aquí & Ssa, ssanit-a, ssanit-in \\
De donde & Zi manis \\
Debajo & Swadday \\
Dentro & Daxer \\
Desde fuera & Zi barra \\
Desde quí, de aquí & Zi ssa \\
Detrás & Deffar, awarni \\
Detrás de mi & Detrás de mi \\
Fuera & barra \\
Hacia adentro & Gar daxer \\
Hasta donde & Ar mani \\
Hasta donde & Ar mani \\
Por debajo & Zi swadday \\
Por dentro & Gar daxer \\
Por detrás & Gar deffar, zi deffar \\
Por donde & Zi manis \\
Por fuera & Zi barra \\
Por/desde/de arriba & Zi sennej \\
\hline & \\
\hline
\end{tabular}


Adverbios de tiempo

\begin{tabular}{|c|c|}
\hline Castellano & Tamazight \\
\hline Ahora & Rexxu, rextu \\
\hline Ahora mismo & Rexxu <ad \\
\hline Al anochecer & Ġar remġạtb \\
\hline Al dia siguiente & Tiwucca nnes \\
\hline Anteayer & faryiḍennaḍ \\
\hline Antes & Adjinna. qber \\
\hline Antes de anteayer & frufaryidẹnnaḍ \\
\hline Aún, todavía & $<$ ad \\
\hline Ayer & Iḍennaḍ \\
\hline Cuando & Mermi, ami \\
\hline De aquí en adelante & Zi ssa gar zzat. zi ssa d tsawent \\
\hline De dia & $S$ wass \\
\hline Desde ahora & Zi rexxu \\
\hline Desde cuando & Zeg wami \\
\hline Desde siempre & Zi rebda \\
\hline $\begin{array}{l}\text { Desde tiempos remotos/ } \\
\text { inmemoriables }\end{array}$ & Zi zman n waman \\
\hline Día/días & Ass/ussan, nhar/nhurat \\
\hline El año pasado & Azgat. asegwas $/<a m$ i ye $<$ dun \\
\hline El próximo año & Imar, asegwas/<am i-d yusin \\
\hline Entonces & Rexdenni \\
\hline Entre hoy y mañana & Jar nhar-a d tiwucca \\
\hline Este año & Asegwas-a, <am-a \\
\hline Estos dias & Ussan-a, riyam-a \\
\hline Hace mucho tiempo & Atțas zeg wami \\
\hline Hace poco tiempo & Drust zeg wami \\
\hline Hace tres años & Trata $n$ isegwusa zeg wami \\
\hline Hoy & Ass-a. ass $n$ yiḍa, nhar-a, nhar $n$ yiḍa \\
\hline Mañana & Tiwucca \\
\hline Noche/noches & Djiret_djiari \\
\hline Nunca & <emmas \\
\hline Para cuando & I mermi \\
\hline Pasado mañana & Farwayecca \\
\hline Pasado pasado mañana & Frufarwayecca \\
\hline Por la mañana & Ġar șbaḥ \\
\hline Por la noche & S djiret. gar tmeddit \\
\hline Por la tarde & $\dot{G a r}$ u<ecci \\
\hline Siempre & Rebda \\
\hline Temprano & Zic \\
\hline
\end{tabular}


Adverbios de modo

\begin{tabular}{|c|c|}
\hline Castellano & Tamazight \\
\hline A caballo & $x$ uyis \\
\hline A escondidas & $S$ tnuffra \\
\hline A gatas & S umuared $x$ iḍaren $\mathbf{d}$ ifassen \\
\hline A la carrera, corriendo & S tazzra \\
\hline A palos & $S$ wu<mud \\
\hline A pie & X iḍaren \\
\hline A prisa & $S$ tazzra, $s$ degiian \\
\hline A tiros & S uqarțas/rexfif/rbaruḍ \\
\hline Al revés & S rmeqrub \\
\hline Así de este/ese modo & Ammu/amenni \\
\hline Asl y asá & Ammu d wammu \\
\hline Como, de que modo & Mamec \\
\hline Con cordura, despacio & $S$ ra<qer \\
\hline Conforme & D wenni d netta \\
\hline De cualquier modo & Mamec ma \\
\hline De hambre & S raz \\
\hline De lado & S ugezdis \\
\hline De pena & S tfeqqaḥt \\
\hline De sed & $S$ fad \\
\hline De verdad & S tidet \\
\hline
\end{tabular}

Adverbios de cantidad

\begin{tabular}{|l|l|}
\hline Castellano & Tamazight \\
\hline Algo & Ca \\
Cuanto & Mecḥar, char \\
De tal tamaño & Anect \\
Demasiado & Aț̣as \\
Igual & Am, kif kif \\
Mucho & Aț̣as \\
Nada & Walu \\
Poco & Drust, cwayt \\
Poquito & Drustit. cwaytit \\
Tanto & Anect \\
\hline
\end{tabular}

Adverbios de afirmación

\begin{tabular}{|l|l|}
\hline Castellano & Tamazight \\
\hline Ciertamente & Stidet \\
Cierto & D wenni d netta \\
De acuerdo & Waxxa, d wenni d netta \\
Es verdad & D tidet \\
$\mathrm{Si}$ & Wah \\
\hline
\end{tabular}


Adverbios de negación y duda

\begin{tabular}{|l|l|}
\hline Castellano & Tamazight \\
\hline Acaso & Ma \\
De ningún modo & $\begin{array}{l}\text { Mamec ma lla, war } \\
\text { xa-s nessiwer }\end{array}$ \\
Imposible (jamás podrá ser) & <emmas adyiriatiri \\
No (para respuesta negativa corta) & Lla \\
No (para respuesta negativa larga) & War \\
Probablemente (puede ser) & Adyiri, atiri \\
Quizá & Balak \\
\hline
\end{tabular}

Pronombres $y$ adverbios interrogativos

\begin{tabular}{|c|c|}
\hline Castellano & Tamazight \\
\hline $\bar{A}$ dónde & Ar mani \\
\hline Cómo & Mamec \\
\hline Con cual & Ak man-wen/man-ten \\
\hline Con cuánto & S mechar \\
\hline Con qué (cuando se cita el objeto) & $S$ man, s mant \\
\hline Con qué (cuando se omite el objeto) & S minzi \\
\hline Con quién & Wik \\
\hline Cual (f.) & Man-ten \\
\hline Cual (m.) & Man-wen \\
\hline Cual de ellas & Man-ten zzay-sent \\
\hline Cual de ellos & Man-wen zzay-sen \\
\hline Cuales $(t)$. & Man-tin \\
\hline Cuales (m.) & Man-yin \\
\hline Cuándo & Mermi \\
\hline Cuánto/s, cuánta/s & Mecḥar, chạ \\
\hline De dónde & $\mathrm{Zi}$ manis \\
\hline De qué & Zi man, zi mant \\
\hline De qué (de qué es) & $\mathrm{N}$ min-t yiran, .......... \\
\hline Desde cuándo & $\mathrm{Zi} \mathrm{mermi}$ \\
\hline Desde dónde & $\mathrm{Zi}$ manis \\
\hline Desde qué & Zi man, zi mant \\
\hline Dónde & Mani \\
\hline Hasta cuándo & Ar mermi \\
\hline Hasta cuánto & Ar mechar /char \\
\hline Hasta dónde & Ar mani \\
\hline Para cuándo & I mermi \\
\hline Para qué & Maimmi \\
\hline Para quién & I umi \\
\hline Por cuánto & $\mathrm{X}$ mechar /cha! \\
\hline Por qué & Maġar \\
\hline Por qué razón & Min xef \\
\hline Qué & Min, man, mant, mana \\
\hline Quién & $W_{i}$ \\
\hline Quiénes & Wi \\
\hline
\end{tabular}




\section{Paula D Angel}

Paula dal talimant, tzeddeg di Munix, kur asegwas ttrạ gar Madrid, minzi ttexs atessiwer taseppaniut. Xemmi ttrah gar Madrid ttiri di taddaṛ $n$ xaris. Xaris n Paula yessawar taseppaniut mlih. Ak tarwa nnes yessawar ḥaca talimant maca xemmi gar-s ttiri Paula yessawar gir taseppaniut.

Angel d aseppaniu, yessawar țrata $n$ yirsawen, maca deg wuxxam ak tarwa nnes yessawar gir taseppaniut. Angel izeddeg di Mlilt, u kur asegwas itraḥ gar Fransa ak rwacum huma tarwa nnes adessiwren tafransist.

\section{Contestad a las siguientes preguntas}

- ¿Min te<na Paula?

- ¿Mamec das eqqaren i tandint mani tzeddeg?

- ¿Maimmi ttrah, kur asegwas, gar Madrid?

- ¿Wi gar-s Paula, zi taǵayamt nnes, di Madrid?

- ¿Mani ttiri, xemmi ttrah gar tandint n igezran n sadu tmuart? 
2. Estableced las preguntas de las siguientes respuestas

$-\dot{c}$ ?

Lla, Paula war tedji bu $\underline{d}$ tafransist, nettat $\underline{d}$ talimant.

$-\dot{c}$

Lla, Angel war yedji bu d agrenzi, netta $\underline{d}$ aseppaniu.

$-\dot{c}$ ?

Netta zi Mlilt, Angel $d$ amlil am neccin.

- ¿

Lla, Angel war yessiwir ca gir taseppaniut, yessawar ura $d$ trata $n$ yirsawen nneg்nit. $-\dot{c}$

Lla, deg uxxam nnes war yessiwir bu tafransist, yessawar gir taseppaniut

3. Construid una oración con cada una de las siguientes palabras

$$
\text { - Asegwas - Tazeddigt }
$$




\section{Agirin i waman}

Agirin i waman war dinni bu raman ${ }^{(2)}$

Agirin i waman war dinni bu raman

War din gar tamara d imețtawen yehman

War din gar tamara d imețtawen yehman

Dinni i remdeg ddedj, dinni i hedmeǵ eccan ${ }^{(2)}$

Dinni i fehmeg $r<e z z$ di tmuart inu i yedjan

Mani tefna tawmat, mani fedren wussan

Mani tefderer tudarat $\underline{d}$ wurawen $n$ yiwdan

Taḍeḥhact urumi d amețta iḥenjaren ${ }^{(2)}$

Essarexsen re<mar di tgarruba ihajaren

Tagarrabut nwem war teqri< di raman

Ticarraw $\underline{d}$ re<zat $i$ bedden $x$ waman

Rami yeqqim Uliman ttarjan- $\underline{\mathrm{t}} \operatorname{siman}^{(2)}$

Adihedoudar re<qer, iya! $<$ ezz wi itgiman

iA! uma regrubiyet am tanda i yaressan

Am tamment i yudumen di rhander yessuar

Wi-t yexsen i tudart $s$ jjennet d tizemmar $^{(2)}$

A-t yerqa d rqudat di re<qaib $n$ zman

iA! uma tammuart inu s wugirum d waman

Xar-ai zi lemnayen $\mathrm{n}$ sqi< Uliman 
Más allá del mar

Más allá del mar no hay seguridad

Más allá del mar no hay seguridad

No hay mas que penurias y lágrimas calientes

No hay mas que penurias y lágrimas calientes

Alli conocí la humillación, allí quebré la altivez

Alli comprendí que la dignidad en mi tierra existía

Donde perecieron mis hermanos, donde los días se ennoblecen

Donde se ennoblece la vida y los corazones de la gente

La sonrisa de Europa es el llanto de los jóvenes

Que desprecian la vida en las pateras herrantes

Vuestra frágil patera no ha partido segura

Los escalofrios y las condolencias de pie están sobre el agua

Con Alemania ya sueñan hasta los bebés

Que tengan presente la cordura y la dignidad los que se quedan

iHermano mío! El desarraigo es como la poza mansa

Como la mezcla de la miel caida sobre la amarga planta

Quien lo desee para su vida con placer y poder

Que lo reciba como obsequio de un destino adverso

iHermano mío! Mi tierra con pan y agua

La prefiero a los millones de las heladas de Alemania 


\begin{tabular}{|c|c|}
\hline Rifenio & castellano \\
\hline 1. Tnayen $\mathrm{n}$ wawmaten, wa war izar wa. Imejjan. & Dos hermanas, una no ve a la otra. Las orejas. \\
\hline 2. Tezwa iǵzar war teggi bu tiri. Tgusyyit. & Cruzó el río y no hizo sombra. El grito. \\
\hline 3. Arb<a n tjiratin war zarrent ay-a uy-a. Tigemmar. & Cuatro vecinas, una no ve a la otra. Las esquinas. \\
\hline $\begin{array}{l}\text { 4. Amșșifeț nnes am isem nnes. Tamellalt /amedjac/. } \\
\text { 5. Ekkin xa-s war yarriz, yiwe, aman yarrez. Rcigeod. }\end{array}$ & $\begin{array}{l}\text { Es igual su descripción que su nombre. El huevo. } \\
\text { Cruzaron sobre él y no se partió y llegó al agua y } \\
\text { se partió. El papel. }\end{array}$ \\
\hline 6. Ḥenna tcețteh. jeddi yebbuțteh. Tadjunt $\underset{d}{ }$ wundu. & $\begin{array}{l}\text { Mi abuela baila y mi abuelo está tumbado. } \\
\text { El cedazo y la panera. }\end{array}$ \\
\hline 7. Bu tiru itnus di barra war ituff. Atmum n urum. & $\begin{array}{l}\text { El ancho pernocta en el descampado y no se moja. } \\
\text { El almiar. }\end{array}$ \\
\hline $\begin{array}{l}\text { 8. Di rexra d izizawen. di ssuq d ibarcanen, } \\
\text { di taddart d izegwagen. Tarjin nig refhem }\end{array}$ & $\begin{array}{l}\text { En el campo están verdes, en mercado negros } \\
\text { y en casa rojos. Las brasas o el carbón. }\end{array}$ \\
\hline 9. Temmut, tessummet. Tahnact & Se murió y posó la cabeza. La viga. \\
\hline 10. Targent inu tejna, tadunt nnes ttmenza. Urțu. & $\begin{array}{l}\text { Mi camella está echada y su sebo se vende. } \\
\text { La higuera. }\end{array}$ \\
\hline 11. Targent inu tejna iri nnes itakkwad ajenna. Tafqunt. & $\begin{array}{l}\text { Mi camella está echada y su cuello alcanza el cielo. } \\
\text { El horno rústico tradicional. }\end{array}$ \\
\hline 12. Tez̧r-ai eẓrig-t. $\mathrm{k}<\mathrm{ejb}-\mathrm{ai}$ esğig-t. Tisit. & Me vió, lo ví, me gustó y lo compré. El espejo. \\
\hline $\begin{array}{l}\text { 13. Ruhen d icemraren, <eqben-d d izegwagen. } \\
\text { inguren. }\end{array}$ & $\begin{array}{l}\text { Partieron blancos, y volvieron dorados. } \\
\text { Las tortas de pán. }\end{array}$ \\
\hline 14. Ttazzer tesrewriw. Taxfift. & Corre gritando alborbolas de alegría. La bala. \\
\hline 15. Tegwar ttbezza< adan nnes. Tisineft. & Camina esparciendo sus intestinos. La aguja. \\
\hline 16. iwḍen igzzar essedhan. Iharkusen. & Al llegar al río se volvieron tímidos. Los zapatos. \\
\hline $\begin{array}{l}\text { 17. Wa ixezzar gar wa, maca ijen war itrih } \\
\text { gar wenneḍ̛nit. Thayen } n \text { idurar. }\end{array}$ & $\begin{array}{l}\text { Los dos se miran mutuamente, pere ninguno } \\
\text { se acerca al otro. Dos montes. }\end{array}$ \\
\hline $\begin{array}{l}\text { 18. Yedden, yesmudden, yedre-d yessecc } \\
\text { midden. Yaziọ }\end{array}$ & $\begin{array}{l}\text { Anunció, reanunció, descendió y dio de comer. } \\
\text { El gallo. }\end{array}$ \\
\hline $\begin{array}{l}\text { 19. Lalla mbra iḍaren tegwar x uziza. treqqeḍ. } \\
\text { teznuza. Tagarrabut. }\end{array}$ & $\begin{array}{l}\text { Mi señora, la sin pies; camina sobre azul. } \\
\text { recoge y vende. La barca. }\end{array}$ \\
\hline $\begin{array}{l}\text { 20. Tazuwda inu n traxt yennd-as-d rizar d aziza. } \\
\text { Tammuart yennọ-as- } d \text { ilel. }\end{array}$ & $\begin{array}{l}\text { Mi plato es de barro y está rodeado de gasa azul. } \\
\text { La tierra rodeada del mar. }\end{array}$ \\
\hline 21. Tessakkwad rexbar maca war tessiwir. Tabrat. & Lleva la noticia pero no habla. La carta. \\
\hline $\begin{array}{l}\text { 22. Yuse-d zeg ij n tmuart war day-s bu yijdi. } \\
\text { garsen-as ij } n \text { icarri war-t yecci war-t yezri. Asiymi. }\end{array}$ & $\begin{array}{l}\text { Vino de una tierra donde no hay arena, le } \\
\text { sacrificaron un cordero que no vió ni comió. } \\
\text { El recién nacido. }\end{array}$ \\
\hline 23. Yecca ar ami yejiwen, yengez $x$ lallas. Aqbuc. & $\begin{array}{l}\text { Comió hasta hartarse y se abalanzó } \\
\text { sobre su señora. El cántaro. }\end{array}$ \\
\hline 24. War-t arebbun isardan, war-t eqqizen irizam. Tin. & $\begin{array}{l}\text { No la portan los mulos ni la cavan los picos. } \\
\text { La sombra. }\end{array}$ \\
\hline 25. Anu $x$ wanu. aman walu. Aganim. & Un pozo sobre otro y agua no hay. La caña. \\
\hline 26. Rkazi i<emmar s yixsan. Agesmar d tigmas. & $\begin{array}{l}\text { Una ventana llena de huesos. } \\
\text { Las mandibulas y los huesos. }\end{array}$ \\
\hline 27. Ġar-s iḍaren war gar-s bu ifassen. Ssamwar. & Tiene pies y no tiene manos. El pantalón. \\
\hline $\begin{array}{l}\text { 28. Gar-s ifassen duzedjif, war gar-s bu iḍaren. } \\
\text { Qubbu. }\end{array}$ & Tiene manos y cabeza pero no tien pies. La chilaba. \\
\hline 29. Ttarra udem gar barra maca war ttwiri. Rkazi. & Da con su rostro al exterior pero no ve. La ventana. \\
\hline $\begin{array}{l}\text { 30. Xemmi-ten tteggeg ttjawanen, } \\
\text { xemmi war-ten tteggeg ttrazan. Iharkusen. }\end{array}$ & $\begin{array}{l}\text { Cuando me los pongo de sacian y cuando no me } \\
\text { los pongo se ponen hambrientos. Los zapatos. }\end{array}$ \\
\hline
\end{tabular}




\begin{tabular}{|llcl|}
\hline & Preterito & Presente intensivo & Futuro \\
\hline Nec & ufíg & ttafé & adafeg \\
Cek & tufid & ttafed & atafed \\
Cem & tufid & ttafed & atafed \\
Netta & yufa & itaf & adyaff \\
Nettat & tufa & ttaf & ataf \\
Neccin & nufa & ntaf & anaf \\
Kenniw & tufim & ttafem & atafem \\
Kennint & tufint & ttafent & atafent \\
Nitnin & ufin & ttafen & adafen \\
Nițenti & ufint & ttafent & adafaent \\
\hline
\end{tabular}

\begin{tabular}{|c|c|c|c|c|c|c|c|}
\hline & \multicolumn{3}{|c|}{ Imperativo precepti } & \multicolumn{4}{|c|}{ Imperativo preceptivo imperfecto } \\
\hline & $\begin{array}{l}\text { cek } \\
\text { cem }\end{array}$ & $\begin{array}{l}\text { afem/afet } \\
\text { afent }\end{array}$ & $\begin{array}{l}\text { kenniw } \\
\text { kennint }\end{array}$ & $\begin{array}{l}\text { ttaf } \\
\text { ttaf }\end{array}$ & $\begin{array}{l}\text { cek } \\
\text { cem }\end{array}$ & $\begin{array}{l}\text { ttafem/ttafe! } \\
\text { ttafent }\end{array}$ & \\
\hline
\end{tabular}

\begin{tabular}{|lll|lll}
\hline \multicolumn{4}{c|}{ Imperativa prohibitivo } & \\
war & ttaf & cek & war & ttafem/ttafet & kenniw \\
war & ttaf & cem & war & ttafent & kennint \\
\hline
\end{tabular}

\begin{tabular}{|c|c|c|c|c|}
\hline \multicolumn{5}{|c|}{ Futuro precedido del interrogativo y la particula preverbal "ga" } \\
\hline ¿Mermi/mani/wik/............ & ga & yafeǵ & nec & ? \\
\hline ¿Mermi/mani/wik/............. & ga & tafed & cek & ? \\
\hline ¿Mermi/mani/wik/............ & ga & tafed & cem & ? \\
\hline ¿Mermi/mani/wik/............. & ga & yaf & netta & ? \\
\hline ¿Mermi/mani/wik/............ & ga & taf & nettat & ? \\
\hline ¿Mermi/mani/wik/............ & ǵa & naf & neccin & ? \\
\hline ¿Mermi/mani/wik/............ & ġa & tafem & kenniw & ? \\
\hline 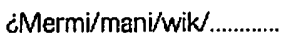 & g̀a & tafent & kennint & $?$ \\
\hline ¿Mermi/mani/wik/............. & g̀a & yafen & nițin & ? \\
\hline ¿Mermi/mani/wik/............ & ga & yafent & nițenti & ? \\
\hline
\end{tabular}

Derivación verbal

Derivación lexical

T(a)wafit: Adivinanza, pl. tiwafitin. 
Verbo amazigh: EXS

Equivalente en castellano: QUERER

\begin{tabular}{|llll|}
\hline & Pretérito & Presente intensivo & Futuro \\
\hline Nec & exseg & ttexseg & adexseg \\
Cek & texsed & ttexsed & atexsed \\
Cem & texsed & ttexsed & atexsed \\
Netta & yexs & itexs & adyexs \\
Nettat & texs & ttexs & atexs \\
Neccin & nexs & ntexs & anexs \\
Kenniw & texsem & ttexsem & atexsem \\
Kennint & texsent & ttexsent & atexsent \\
Nitnin & exsen & ttexsen & adexsen \\
Nitenti & exsent & ttexsent & 8dexsent \\
\hline
\end{tabular}

\begin{tabular}{|c|c|c|c|c|c|c|c|}
\hline \multicolumn{4}{|c|}{ Imperativo perfecto preceptivo } & \multicolumn{3}{|c|}{ Imperativo imperfecto intensivo } & \\
\hline $\begin{array}{l}\text { exs } \\
\text { exs }\end{array}$ & $\begin{array}{l}\text { cek } \\
\text { cem }\end{array}$ & $\begin{array}{l}\text { exsem/exset } \\
\text { exsent }\end{array}$ & $\begin{array}{l}\text { kenniw } \\
\text { kennint }\end{array}$ & $\begin{array}{l}\text { thexs } \\
\text { ttexs }\end{array}$ & $\begin{array}{l}\text { cek } \\
\text { cem }\end{array}$ & $\begin{array}{l}\text { ttexsem/ttexset } \\
\text { ttexsent }\end{array}$ & $\begin{array}{l}\text { kenniw } \\
\text { kennint }\end{array}$ \\
\hline
\end{tabular}

\begin{tabular}{|lll|lll|}
\hline \multicolumn{5}{|c|}{ Imperativo prohibinivo } \\
\hline war & ttexs & cek & war & ttexsem/ttexset & kenniw \\
war & ttexs & cem & war & ttexsent & kennint \\
\hline
\end{tabular}

\begin{tabular}{|c|c|c|c|c|}
\hline \multicolumn{5}{|c|}{ Futuro precedido del Interrogativo y la partscuta preverbal "ga" } \\
\hline 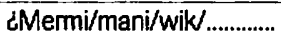 & ga & exseg & nec & $?$ \\
\hline ¿Mermi/mani/wik/............. & g̀a & texsed & cek & ? \\
\hline ¿Mermi/mani/wik/............. & g̀a & texsed & cem & ? \\
\hline LMermi/mani/wik/.............. & ga & yexs & netta & ? \\
\hline ¿Mermi/mani/wik/............. & $\dot{\mathrm{g} a}$ & texs & nettat & ? \\
\hline ¿Mermi/mani/wik/............ & ga & nexs & neccin & ? \\
\hline 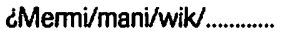 & ga & texsem & kenniw & ? \\
\hline ¿Mermi/mani/wik/.............. & g̀a & texsent & kennint & ? \\
\hline ¿Mermi/mani/wik/............. & ga & exsen & niţnin & ? \\
\hline ¿Mermi/mani/wik/............. & g̀a & exsent & nitenti & ? \\
\hline
\end{tabular}

Derivación verbal

Derivación lexica!

Timexsiwt: deseo: pl. timexsiwin. 
Verbo amazigh: IRI [ILI] Equivalente en castellano: SER, ESTAR y HABER

\begin{tabular}{|llcl|}
\hline & Preterrito & Presente intensivo & Futuro \\
\hline Nec & edjig & ttirig & adirig \\
Cek & tedjid & ttirid & atirid \\
Cem & tedjid & ttirid & atirid \\
Netta & yedja & itiri & adyiri \\
Nettat & tedja & ttiri & atiri \\
Neccin & nedja & ntiri & aniri \\
Kenniw & tedjam & ttirim & atirim \\
Kennint & tedjant & ttirint & atirint \\
Nițin & edjan & ttirin & adirin \\
Nițenti & edjant & ttirint & adirint \\
\hline
\end{tabular}

\begin{tabular}{|c|c|c|c|c|c|c|c|}
\hline \multicolumn{4}{|c|}{ Imperativo perfecto preceptivo } & \multicolumn{4}{|c|}{ Imperativo imperfecto intensivo } \\
\hline iri & $\begin{array}{l}\text { cek } \\
\text { cem }\end{array}$ & $\begin{array}{l}\text { irim/irit } \\
\text { irint }\end{array}$ & $\begin{array}{l}\text { kenniw } \\
\text { kennint }\end{array}$ & $\begin{array}{l}\text { ttiri } \\
\text { ttiri }\end{array}$ & $\begin{array}{l}\text { cek } \\
\text { cem }\end{array}$ & $\begin{array}{l}\text { ttirim/ttirit } \\
\text { ttirint }\end{array}$ & $\begin{array}{l}\text { kenniw } \\
\text { kennint }\end{array}$ \\
\hline
\end{tabular}

\begin{tabular}{|lll|lll|}
\hline \multicolumn{4}{|c|}{ Imperativo prohibitivo } & \\
\hline war & ttiri & cek & war & ttirim/ttirit & kenniw \\
war & ttiri & cem & war & ttirint & kennint \\
\hline
\end{tabular}

\begin{tabular}{|c|c|c|c|c|}
\hline \multicolumn{5}{|c|}{ Futuro precedido del interrogativo y la particula preverbal "ga" } \\
\hline ¿Mermi/mani/wik/........... & $\dot{\mathrm{g} a}$ & irig & nec & ? \\
\hline ¿Mermi/mani/wik/.............. & ǵa & tirid & cek & ? \\
\hline ¿Mermi/mani/wik/.............. & ga & tirid & cem & ? \\
\hline ¿Mermi/mani/wik/.............. & ǵa & yiri & netta & $?$ \\
\hline ¿Mermi/mani/wik/.................... & g̀a & tiri & nettat & ? \\
\hline ¿Mermi/mani/wik/.................. & g̀a & niri & neccin & $?$ \\
\hline 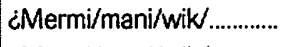 & ga & tirim & kenniw & ? \\
\hline ¿Mermi/mani/wik/............. & $\dot{\mathrm{g} a}$ & tirint & kennint & ? \\
\hline ¿Mermi/mani/wik/................ & ġa & irin & nițnin & ? \\
\hline ¿Mermi/mani/wik/............. & $\dot{g} \mathbf{a}$ & irint & nitenti & ? \\
\hline
\end{tabular}

Derivación verbal

Derivación lexical 
Vocabulario

\begin{tabular}{|c|c|c|c|c|c|}
\hline \multirow[t]{2}{*}{ Colores } & \multicolumn{2}{|c|}{ Masculino } & \multicolumn{2}{|c|}{ Femenino } & \multirow[t]{2}{*}{ Observaciones } \\
\hline & Singutar & Plural & Singutar & Pural & \\
\hline Amarillo & awrag & iwragen & tawragt & tiwragin & \\
\hline $\begin{array}{l}\text { Apagado, flojo, } \\
\text { deshinchado }\end{array}$ & yexsi & xesien & texsi & xesient & $\begin{array}{l}\text { Adj. v. de } x s i=\text { apagar(se), } \\
\text { deshinchar(se). Para la } 3^{\mathrm{a}} \text { persona }\end{array}$ \\
\hline Azul & azbaybiy & izbaybiyen & tazbaybiyt & tizbaybiyin & ${ }^{*}(\mathrm{iyt})=/ \mathrm{ect} /$ \\
\hline Blanco & $\begin{array}{l}\text { acemrar } \\
\text { amlil } \\
\text { amellal }\end{array}$ & $\begin{array}{l}\text { icemraren } \\
\text { imlilen } \\
\text { imellalen }\end{array}$ & $\begin{array}{l}\text { tacemrart } \\
\text { tamlilt } \\
\text { tamellalt }\end{array}$ & $\begin{array}{l}\text { ticemrarin } \\
\text { timlilin } \\
\text { timellalin }\end{array}$ & $\begin{array}{l}\text { El origen es acemlal, pero en } \\
\text { Guelaya tas is se transforman } \\
\text { en } r \text {. habitualmente }\end{array}$ \\
\hline Castaño & azumbiy & izumbiyen & tazumbiyt* & tizumbiyin & Literalmente: panocha. * (iyt)=/ect/ \\
\hline $\begin{array}{l}\text { Claso, } \\
\text { encendido }\end{array}$ & $\begin{array}{l}\text { yareqq } \\
\text { yargáa }\end{array}$ & $\begin{array}{l}\text { areqqen } \\
\text { argin }\end{array}$ & $\begin{array}{l}\text { tareqq } \\
\text { targa }\end{array}$ & $\begin{array}{l}\text { areqqent } \\
\text { argint }\end{array}$ & $\begin{array}{l}\text { Adj. v. de } \arg =\text { encender(se) } \\
\text { Para la } 3^{\mathrm{a}} \text { persona }\end{array}$ \\
\hline Descolorido & yekcef & kecfen & tekcef & kecfent & $\begin{array}{l}\text { Adj. v. de } k c e f=\text { deslucir(se). el v. } \\
\text { denota: desdicha, infortunio, miseria, } \\
\text { frustración,etc. Para la } 3^{a} \text { persona }\end{array}$ \\
\hline Estampado & yarcem & arcmen & tarcem & arcment & $\begin{array}{l}\text { Adj. v. de arcem = estampar, } \\
\text { marcar, señalar. Para la } 3^{\mathrm{B}} \text { persona }\end{array}$ \\
\hline Marrón & aqehwiy & iqehwiyen & taqehwiyt & tiqehwiyin & $\begin{array}{l}\text { De la pl. árabe qahwa = café. } \\
\text { "(iyt)=/ect/ }\end{array}$ \\
\hline Moteado & ineqqed & neqqden & tneqqed & neqqdent & $\begin{array}{l}\text { Adj.v. de aneqqiḍ -taneqqit. } \\
\text { (dt) }=/ t / \text { : gota, del árabe nuq.a. } \\
\text { Para la } 3^{\mathrm{a}} \text { persona }\end{array}$ \\
\hline Negro & abarcan & ibarcanen & tabarcant & tibarcanin & \\
\hline Oscuro & yeḥdeq & hedquen & tehọeq & hedqent & Adj. v. Para la $3^{\mathrm{a}}$ persona \\
\hline Pelirojo & azumbiy & izumbiyen & tazumbiyt* & tizumbiyin & ${ }^{*}(\mathrm{iyt})=/ \mathrm{ect}$ \\
\hline Rayado, listado & icarḍ & carden & tcard & cardent & $\begin{array}{l}\text { Adj.v. de carred }=\text { rayar, acarrid }= \\
\text { raya. Para la } 3^{\mathrm{a}} \text { persona }\end{array}$ \\
\hline Rojo & azegwaǵ & izegwaǵen & tazegwag̀t & tizegwagin & \\
\hline Rosa, rosado & awardiy & iwardiyen & tawardiyt & tiwardiyin & $*(\mathrm{iyt})=/ \mathrm{ect} /$ \\
\hline Rubio & acuwraq & icuwraqen & tacuwraqt & ticuwraqin & \\
\hline Verde & aziza & izizawen & tazizawt & tizizawin & \\
\hline
\end{tabular}




\section{Vocabulario}

\begin{tabular}{|c|c|c|c|c|c|}
\hline \multirow[t]{2}{*}{$\begin{array}{l}\text { Objetos y utensilias } \\
\text { domésticos }\end{array}$} & \multicolumn{2}{|c|}{ Masculino } & \multicolumn{2}{|c|}{ Femenino } & \multirow{2}{*}{ Observaciones } \\
\hline & Singular & Plural & Singular & Plural & \\
\hline Aceitera & & & taziyyat & tiziyyațin & $\begin{array}{l}\text { Del ár. zayt, aceite. } \\
\text { Botella comúnmente }\end{array}$ \\
\hline Aguja & isiyni & isiynan & $\begin{array}{l}\text { tisiynit } \\
\text { tisineft }\end{array}$ & $\begin{array}{l}\text { tisiynan } \\
\text { tisinaf }\end{array}$ & $\begin{array}{l}\text { El m. es superlativo: (aguja de } \\
\text { hacer punto) p. ej. }\end{array}$ \\
\hline Almirez, mortero & $\begin{array}{l}\text { amehraz } \\
\text { rmehraz }\end{array}$ & $\begin{array}{l}\text { imehrazen } \\
\text { rmehrazat }\end{array}$ & & & Del ár. al-mihraz \\
\hline $\begin{array}{l}\text { Ánfora, cántaro } \\
\text { de dos asas }\end{array}$ & aqbuc & iqubac & taqbuct & tiqubac & $\begin{array}{l}\text { El f. es diminutivo. Agraw } n \text { wugbuc } \\
=\text { el asa del cántaro }\end{array}$ \\
\hline Azucarero & & & $\begin{array}{l}\text { tarbi<t n } \\
\text { ssekkwar }\end{array}$ & $\begin{array}{l}\text { tarbi<in } n \\
\text { ssekkwar }\end{array}$ & \\
\hline $\begin{array}{l}\text { Balde, cubo, } \\
\text { bidón }\end{array}$ & $\begin{array}{l}\text { abidaw } \\
\text { rbidu } \\
\text { abidun }\end{array}$ & $\begin{array}{l}\text { ibidawen } \\
\text { rbadawat } \\
\text { ibidunen }\end{array}$ & tabidawt & tibidawin & Del fr. bidon \\
\hline Bandeja & & & ssiniyet & sswani & Ssinivet $n$ watay = bandeja de té \\
\hline Bocal, pote, tarro & $\begin{array}{l}\text { azembir } \\
\text { aqdih }\end{array}$ & $\begin{array}{l}\text { izembiren } \\
\text { iqdihen }\end{array}$ & taqdiht & tiqdihin & $\begin{array}{l}\text { Azembir } n \text { watay }=\text { estuche del té. } \\
\text { Aqdih (taziyyat) } n \text { tamment }= \\
\text { tarro de miel }\end{array}$ \\
\hline $\begin{array}{l}\text { Bolsa o saco } \\
\text { de plástico }\end{array}$ & & & $\begin{array}{l}\mathrm{t}[\text { a]xancet } n \\
\text { plastik }\end{array}$ & $\begin{array}{l}\text { tixuncay } \\
\text { n plastik }\end{array}$ & \\
\hline $\begin{array}{l}\text { Bombona } \\
\text { de gas }\end{array}$ & & & $\begin{array}{l}\text { taziyyat } n \\
\text { buțano }\end{array}$ & $\begin{array}{l}\text { tiziyyațin } n \\
\text { buțano }\end{array}$ & También taziyyat n lgaz \\
\hline Botella & & & taziyat & tiziyatin & \\
\hline Cafetera & agellay & iǵellayen & taǵellayt & tigellayin & $\begin{array}{l}\text { Del ár. gala, galayan: hervir. } \\
\text { hervimiento. El m. es superlativo }\end{array}$ \\
\hline $\begin{array}{l}\text { Calentador } \\
\text { de agua }\end{array}$ & asehmay & iseḥmayen & & & $\begin{array}{l}\text { Del v. transitivo amaz. Sihma: } \\
\text { calentar, del ref. hma; calentar(se). }\end{array}$ \\
\hline Cesta, canasta & & & $\mid \begin{array}{l}\text { taqrabt } \\
\mathrm{t} \text { (a)suddiyt* } \\
\text { / tsuddct } /\end{array}$ & $\begin{array}{l}\text { tiqurab } \\
\text { tisuddiyin }\end{array}$ & $\begin{array}{l}\text { Tiqurab se elaboran de esparto (ari) } \\
\text { y tisuddiyin de caña (ganim) } \\
\text { o mimbre. * (iyt)=/ect }\end{array}$ \\
\hline $\begin{array}{l}\text { Cobertera, tapa, } \\
\text { tapadera, tapón }\end{array}$ & aqeffar & iqeffaren & \begin{tabular}{|l} 
taqebbart \\
taqeffart \\
$/$ taqeffac /
\end{tabular} & $\begin{array}{l}\text { tiqebbarin } \\
\text { tiqeffarin }\end{array}$ & $\begin{array}{l}\text { Taqebbart } n \text { ssenduq. Aqeffar } n \\
\text { wuqbuc. Taqeffac } n \text { tziyyat }\end{array}$ \\
\hline
\end{tabular}

